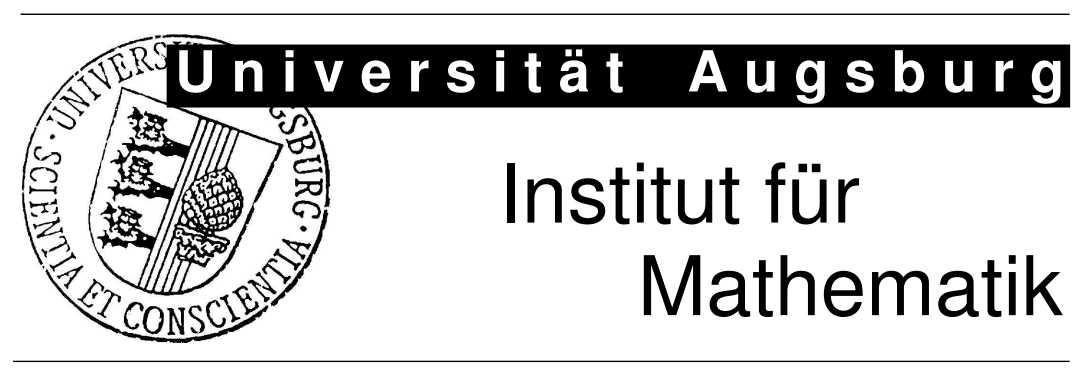

Christoph Kawan

Lower Bounds for the Strict Invariance Entropy

Preprint Nr. 10/2010 - 30. Juli 2010

Institut für Mathematik, Universitätsstraße, D-86135 Augsburg

http: //www.math. uni-augsburg.de/ 


\section{Impressum:}

\section{Herausgeber:}

Institut für Mathematik

Universität Augsburg

86135 Augsburg

http://www.math.uni-augsburg.de/pages/de/forschung/preprints.shtml

\section{ViSdP:}

Christoph Kawan

Institut für Mathematik

Universität Augsburg

86135 Augsburg

Preprint: Sämtliche Rechte verbleiben den Autoren (C) 2010 


\title{
Lower bounds for the strict invariance entropy
}

\author{
C Kawan \\ Institut für Mathematik, Universität Augsburg, 86135 Augsburg/Germany \\ E-mail: christoph.kawan@math.uni-augsburg.de
}

\begin{abstract}
In this paper, we present a new method for obtaining lower bounds of the strict invariance entropy by combining an approach from the theory of escape rates and geometric methods used in the dimension theory of dynamical systems. For uniformly expanding systems and for inhomogeneous bilinear systems we can describe the lower bounds in terms of certain uniform volume growth rates. In particular, we obtain criteria for positive entropy.
\end{abstract}

AMS classification scheme numbers: 93C15, 34C40, 94A17

\section{Introduction}

Invariance entropy for continuous-time control systems was introduced in [2] to measure how often open-loop control functions have to be updated in order to achieve invariance of a given compact and controlled invariant subset $Q$ of the state space for a fixed set of initial states $K \subset Q$. For $K=Q$ it is shown in [9] that the (strict) invariance entropy $h_{\mathrm{inv}}^{*}(Q)$ equals the minimal data rate in a feedback loop necessary to render the set $Q$ invariant by a causal coding and control law. General upper and lower bounds were established in [8]. In [10], we derived an upper estimate for the strict invariance entropy of a control set in terms of the Lyapunov exponents of a periodic solution. In the present paper, we use a new approach to derive sharper lower bounds for the strict invariance entropy $h_{\mathrm{inv}}^{*}(K, Q)$, which is based on the observation that $h_{\mathrm{inv}}^{*}(K, Q)$ is bounded from below by a quantity that is very similar to the escape rates of dynamical systems, studied, e.g., in [13, 14]. For a dynamical system, given by a map $f: X \circlearrowleft$ on a compact metric space $(X, d)$, the (upper) escape rate from a set $Q \subset X$ is defined by

$$
\lambda=\lambda(f, m, Q):=\limsup _{n \rightarrow \infty} \frac{1}{n} \log m\left(\bigcap_{i=0}^{n-1} f^{-i}(Q)\right),
$$

where $m$ denotes a reference measure on $X$. By covering the sets $\bigcap_{i=0}^{n-1} f^{-i}(Q)$ with Bowen-balls

$$
B^{n}(x, \varepsilon)=\left\{y \in X: d\left(f^{i}(x), f^{i}(y)\right)<\varepsilon, i=0,1, \ldots, n-1\right\},
$$


centred at the elements $x$ of maximal $(n, \varepsilon, f)$-separated subsets, and applying standard arguments from the proof of the variational principle for pressure, one obtains an $f$ invariant measure $\mu$ supported on $Q$ such that

$$
\lambda \leq P_{\mu}(f,-\varphi)=h_{\mu}(f)-\int \varphi d \mu
$$

holds for any continuous function $\varphi: X \rightarrow \mathbb{R}$ that satisfies

$$
m\left(B^{n}(x, \varepsilon)\right) \leq C \mathrm{e}^{-\sum_{i=0}^{n-1} \varphi\left(f^{i}(x)\right)} .
$$

For a control system $\dot{x}=F(x, u), u \in \mathcal{U}$, on a smooth manifold $M$ with solution maps $\varphi_{t, u}: M \rightarrow M$ it follows easily from the definition of $h_{\text {inv }}^{*}(K, Q)$ that

$$
h_{\text {inv }}^{*}(K, Q) \geq-\limsup _{\tau \rightarrow \infty} \frac{1}{\tau} \ln \sup _{u \in \mathcal{U}} m\left(K \cap \bigcap_{t \in[0, \tau]} \varphi_{t, u}^{-1}(Q)\right)
$$

for any Borel measure $m$ on $M$ such that $0<m(K)<\infty$. Since the control system induces nonautonomous dynamical systems $\left(\varphi_{t, u}\right)_{t \in \mathbb{R}}(u \in \mathcal{U})$ on $M$, the concept of invariant measures does not apply here. Instead, we use a technique from the dimension theory of dynamical systems which allows to estimate the volumes of Bowen-balls by singular value functions of the tangent mappings of the given dynamical systems. Here we use a result of Katrin Gelfert [6, 7], which can be generalized from the autonomous to the nonautonomous context very easily. We end up with a lower bound for $h_{\text {inv }}^{*}(K, Q)$, which splits into two additive parts that resemble the summands of the right-hand side in (1). One of them describes in some sense the "escape behavior" of the system from the set $Q$, the other one describes the minimal exponential growth rate of $n$-dimensional volumes on a given ( $n$-dimensional) subbundle of the extended state space.

The paper is structured as follows: In the second section, we recall basic facts on Hausdorff and capacitive measure, nonlinear control systems, singular value functions and ellipsoids in Euclidean space. We also introduce the notion of strict invariance entropy and some new quantities related to invariance entropy, which will be used for the proof of our main theorem. In Section 3, we adapt Katrin Gelfert's lemma on estimating the Hausdorff measure of Bowen-balls to control systems. The main result, Theorem 4.1, is proved in Section 4. Finally, in Sections 5 and 6, we apply our estimate to uniformly expanding systems and to inhomogeneous bilinear systems, respectively. In both cases, our general lower bound reduces to a uniform volume growth rate. In particular, we are able to show that under the assumption of $K$ having positive volume, the strict invariance entropy $h_{\text {inv }}^{*}(K, Q)$ is positive for a system which is uniformly expanding on $Q$. For an inhomogeneous bilinear system, the same can be shown under the assumption that the associated homogeneous system is expanding on a nontrivial invariant subbundle for the corresponding control flow. 


\section{Preliminaries}

\subsection{Notation}

The term "smooth" always stands for $C^{\infty}$. By a smooth manifold we mean a connected, second-countable, topological Hausdorff manifold endowed with a smooth atlas. If $M$ is a smooth manifold, we write $T_{x} M$ for the tangent space at $x \in M$. The zero element of $T_{x} M$ is denoted by $0_{x}$. We write $T_{Q} M$ for the tangent bundle over a subset $Q$ of $M$ and $T M$ for $T_{M} M$. The derivative of a $C^{1}$-mapping $\phi: M \rightarrow N$ at $x \in M$ is denoted by $D \phi(x): T_{x} M \rightarrow T_{\phi(x)} N$. A diffeomorphism $\phi: M \rightarrow N$ is a smooth invertible mapping such that also $\phi^{-1}: N \rightarrow M$ is smooth. A Riemannian metric on a smooth manifold $M$ is a smooth function $g$ which assigns to each $x \in M$ an inner product $g_{x}$ on $T_{x} M$. The norm induced by $g_{x}$ is denoted by $\|\cdot\|_{T_{x} M}$. For a Riemannian manifold $(M, g)$, the Riemannian exponential map at $x \in M$ is denoted by $\exp _{x}$. We write $B_{\varepsilon}(x)$ for the open ball of radius $\varepsilon$ centred at $x \in M$. By $N_{\varepsilon}(A)$ we denote the open $\varepsilon$-neighbourhood of a set $A$.

By $\lfloor d\rfloor$ we denote the greatest integer strictly smaller than $d \in \mathbb{R}$. For the number of elements in a finite set $A$ we write $\# A$.

\subsection{Hausdorff and capacitive measure}

Let $(X, \varrho)$ be a metric space, $Z \subset X$, and $d \geq 0, \varepsilon>0$. Define

$$
\mu_{H}(Z, d, \varepsilon)=\mu_{H}(Z, d, \varepsilon ; \varrho):=\inf \left\{\sum_{j \geq 1} r_{j}^{d}: r_{j} \leq \varepsilon, Z \subset \bigcup_{j \geq 1} B_{r_{j}}\right\},
$$

where the infimum is taken over all countable covers of $Z$ by balls $B_{r_{j}}$ of radii $r_{j} \leq \varepsilon$. The function $\mu_{H}(\cdot, d, \varepsilon)$ is an outer measure on $X$. For fixed $Z$ and $d$ the function $\mu_{H}(Z, d, \cdot)$ does not decrease with decreasing $\varepsilon$ and hence the limit

$$
\mu_{H}(Z, d)=\mu_{H}(Z, d ; \varrho):=\lim _{\varepsilon \searrow 0} \mu_{H}(Z, d, \varepsilon)=\sup _{\varepsilon>0} \mu_{H}(Z, d, \varepsilon)
$$

exists (it may be $\infty) . \mu_{H}(Z, d)$ is called the $d$-dimensional outer Hausdorff measure of $Z$. The function $\mu_{H}(\cdot, d)$ is a metric outer measure on $X$ (i.e., the restriction of $\mu_{H}(\cdot, d)$ to the Borel- $\sigma$-algebra of $X$ is a measure).

For $Z \subset X, d \geq 0$ and $\varepsilon>0$ we also introduce the quantity

$$
\mu_{C}(Z, d, \varepsilon)=\mu_{C}(Z, d, \varepsilon ; \varrho):=\varepsilon^{d} N_{\varepsilon}(Z),
$$

where $N_{\varepsilon}(Z)$ is the minimal number of $\varepsilon$-balls necessary to cover $Z$ (cp. also [1, Ch. III, Sec. 2.2])). It is easy to see that $\mu_{H}(Z, d, \varepsilon) \leq \mu_{C}(Z, d, \varepsilon)$. We define

$$
\mu_{C}(Z, d)=\mu_{C}(K, d ; \varrho):=\limsup _{\varepsilon \searrow 0} \mu_{C}(Z, d, \varepsilon) .
$$

The properties of $\mu_{C}(Z, d)$ are similar to those of $\mu_{H}(Z, d)$. In particular, $\mu_{C}(\cdot, d, \varepsilon)$ and $\mu_{C}(\cdot, d)$ are outer measures on $X$. 


\subsection{Control systems}

Let $(M, g)$ be a $d$-dimensional Riemannian manifold and

$$
\dot{x}(t)=F(x(t), u(t)), \quad u \in \mathcal{U},
$$

a control system on $M$ with continuous right-hand side $F: M \times \mathbb{R}^{m} \rightarrow T M$ satisfying $F(x, u) \in T_{x} M$, smooth in the first argument. The set of admissible control functions is given by

$$
\mathcal{U}=\left\{u: \mathbb{R} \rightarrow \mathbb{R}^{m}: u \text { measurable with } u(t) \in U \text { a.e. }\right\},
$$

where $U \subset \mathbb{R}^{m}$ is compact. By these assumptions, for every control function $u \in \mathcal{U}$ and every initial value $x \in M$ a unique solution $\varphi(t, x, u)$ satisfying $\varphi(0, x, u)=x$ exists. Additionally, we assume that this solution is defined for all $t \in \mathbb{R}$. If we want to view the solution as a function of the initial value, we write $\varphi_{t, u}(x)$ instead of $\varphi(t, x, u)$. By smoothness of $F$ in the first argument, the maps $\varphi_{t, u}: M \rightarrow M$ are diffeomorphisms. For each $u \in U$, the vector field $x \mapsto F(x, u)$ will also be denoted by $F_{u}$. On $\mathcal{U}$ we introduce the shift flow

$$
\Theta: \mathbb{R} \times \mathcal{U} \rightarrow \mathcal{U}, \quad\left(\Theta_{t} u\right)(s) \equiv u(t+s) .
$$

Note that we do not need continuity of $\Theta$ with respect to any topology on $\mathcal{U}$ in order to prove our main result. In Section 6 , we will endow $\mathcal{U}$ with a weak*-topology which makes it a compact metrizable space and the shift flow a continuous dynamical system, which works for control-affine systems with compact and convex control range. The mapping $\varphi: \mathbb{R} \times M \times \mathcal{U} \rightarrow M$ satisfies the cocycle property

$$
\varphi\left(t, \varphi(s, x, u), \Theta_{s} u\right) \equiv \varphi(t+s, x, u) \text { for all } t, s \in \mathbb{R}, \quad(u, x) \in \mathcal{U} \times M .
$$

Let $Q \subset M$ be a compact controlled invariant set, i.e., for every $x \in Q$ there is $u \in \mathcal{U}$ with $\varphi\left(\mathbb{R}_{0}^{+}, x, u\right) \subset Q$. Then for each compact set $K \subset Q$ the strict invariance entropy $h_{\mathrm{inv}}^{*}(K, Q)$ is defined as follows: A set $\mathcal{S}^{*} \subset \mathcal{U}$ is called $(\tau, K, Q)$-spanning if for every $x \in K$ there is $u \in \mathcal{S}^{*}$ such that $\varphi(t, x, u) \in Q$ for all $t \in[0, \tau]$. By $r_{\text {inv }}^{*}(\tau, K, Q)$ we denote the minimal cardinality of such a set and we define

$$
h_{\mathrm{inv}}^{*}(K, Q):=\limsup _{\tau \rightarrow \infty} \frac{1}{\tau} \ln r_{\mathrm{inv}}^{*}(\tau, K, Q) .
$$

We further define the lift of $Q$ to $\mathcal{U} \times M$ by

$$
\mathcal{Q}:=\left\{(u, x) \in \mathcal{U} \times M: \varphi\left(\mathbb{R}_{0}^{+}, x, u\right) \subset Q\right\} .
$$

With the set $\mathcal{Q}$ we can associate a vector bundle of $\operatorname{rank} d=\operatorname{dim} M$ :

$$
\pi_{\mathcal{Q}}: \bigcup_{(u, x) \in \mathcal{Q}}\{u\} \times T_{x} M \rightarrow \mathcal{Q}, \quad \pi_{\mathcal{Q}}(u, v)=(u, x) \text { if } v \in T_{x} M .
$$

On $\mathcal{U}$ we may consider the natural topology from $L^{\infty}\left(\mathbb{R}, \mathbb{R}^{m}\right)$, which makes $\mathcal{Q} \subset \mathcal{U} \times M$ a metrizable space. The vector space structure on the fibres $\{u\} \times T_{x} M=\pi_{\mathcal{Q}}^{-1}(u, x)$ is the natural one induced by the vector space structure of $T_{x} M$. 
By $\pi_{\mathcal{U}}: \mathcal{U} \times M \rightarrow \mathcal{U}$ we denote the projection onto the first factor, $\pi_{\mathcal{U}}(u, x)=u$. For each compact set $K \subset Q$ we define

$$
\mathcal{K}_{Q}:=\{(u, x) \in \mathcal{Q}: x \in K\} .
$$

Moreover, for each $u \in \pi_{\mathcal{U}} \mathcal{K}_{Q}$ we introduce the nonempty compact sets

$$
K(u, \tau):=\{x \in K: \varphi(t, x, u) \in Q \text { for all } t \in[0, \tau]\}, \quad \tau>0 .
$$

Let $\varrho$ denote the geodesic distance induced by the Riemannian metric $g$. Then for each $u \in \mathcal{U}$ and $\tau>0$ we define the Bowen-metric

$$
\varrho_{u, \tau}(x, y):=\max _{t \in[0, \tau]} \varrho(\varphi(t, x, u), \varphi(t, y, u)) .
$$

It is easy to see that $\varrho_{u, \tau}$ indeed is a metric on $M$ which is topologically equivalent to $\varrho$. (See also [11], where topological entropy is defined for nonautonomous dynamical systems.) For each $(u, x) \in \mathcal{U} \times M, \tau>0$, and $\varepsilon>0$ we define the Bowen-ball

$$
B_{\varepsilon}^{\tau}(u, x):=\left\{y \in M: \varrho_{u, \tau}(x, y)<\varepsilon\right\} .
$$

A set $S \subset M$ is called $(u, \tau, \varepsilon)$-separated if for all $x_{1}, x_{2} \in S$ with $x_{1} \neq x_{2}$ one has $\varrho_{u, \tau}\left(x_{1}, x_{2}\right) \geq \varepsilon$. By $r_{\text {sep }}(u, \tau, \varepsilon, K, Q)$ we denote the maximal cardinality of a $(u, \tau, \varepsilon)$ separated subset of $K(u, \tau)$. We say that a set $F \subset M(u, \tau, \varepsilon)$-spans another set $G \subset M$ if for every $x \in G$ there is $y \in F$ such that $\varrho_{u, \tau}(x, y)<\varepsilon$. By $r_{\text {span }}(u, \tau, \varepsilon, K, Q)$ we denote the minimal cardinality of a set which $(u, \tau, \varepsilon)$-spans $K(u, \tau)$. It is easy to see that a maximal $(u, \tau, \varepsilon)$-separated subset $S$ of $K(u, \tau)$ also $(u, \tau, \varepsilon)$-spans $K(u, \tau)$ (cp. proof of Prop. 2.1 (i)) and hence it holds that

$$
K(u, \tau) \subset \bigcup_{x \in S} B_{\varepsilon}^{\tau}(u, x) .
$$

In addition, we call a set $S \subset M \varepsilon$-separated if $\varrho\left(x_{1}, x_{2}\right) \geq \varepsilon$ holds for each pair of distinct points $x_{1}, x_{2} \in S$. We define

$$
\begin{aligned}
\bar{r}_{\mathrm{sep}}(\tau, \varepsilon, K, Q) & :=\sup _{u \in \pi_{\mathcal{U}} \mathcal{K}_{Q}} \varepsilon^{d} r_{\mathrm{sep}}(u, \tau, \varepsilon, K, Q), \\
\bar{r}_{\mathrm{sep}}(\tau, K, Q) & :=\limsup _{\varepsilon \searrow 0} \bar{r}_{\mathrm{sep}}(\tau, \varepsilon, K, Q), \\
h_{\mathrm{esc}}(K, Q) & =h_{\mathrm{esc}}(K, Q ; g):=\limsup _{\tau \rightarrow \infty} \frac{1}{\tau} \ln \bar{r}_{\mathrm{sep}}(\tau, K, Q) .
\end{aligned}
$$

By definition, $h_{\mathrm{esc}}(K, Q)$ is an element of the extended real line $[-\infty, \infty]$, and it might depend on the Riemannian metric $g$. As for the topological entropy, one obtains an alternative definition of $h_{\text {esc }}(K, Q)$ by replacing maximal $(u, \tau, \varepsilon)$-separated subsets of $K(u, \tau)$ by minimal $(u, \tau, \varepsilon)$-spanning sets (cp. Prop. 2.1(ii)). We define

$$
\begin{aligned}
& \bar{r}_{\text {span }}(\tau, \varepsilon, K, Q):=\sup _{u \in \pi_{\mathcal{U}} \mathcal{K}_{Q}} \varepsilon^{d} r_{\text {span }}(u, \tau, \varepsilon, K, Q), \\
& \bar{r}_{\text {span }}(\tau, K, Q):=\limsup _{\varepsilon \searrow 0} \bar{r}_{\text {span }}(\tau, \varepsilon, K, Q) .
\end{aligned}
$$

Then we can prove the following statements: 
(i) $r_{\mathrm{span}}(u, \tau, \varepsilon, K, Q) \leq r_{\mathrm{sep}}(u, \tau, \varepsilon, K, Q) \leq r_{\mathrm{span}}(u, \tau, \varepsilon / 2, K, Q)<\infty$.

(ii) $h_{\mathrm{esc}}(K, Q)=\lim \sup _{\tau \rightarrow \infty}(1 / \tau) \ln \bar{r}_{\mathrm{span}}(\tau, K, Q)$.

(iii) If $g$ and $\widetilde{g}$ are complete Riemannian metrics on $M$, then

$$
h_{\mathrm{esc}}(K, Q ; g)=h_{\mathrm{esc}}(K, Q ; \widetilde{g}) .
$$

(iv) $h_{\mathrm{esc}}(K, Q)<\infty$.

(v) If $h_{\mathrm{inv}}^{*}(Q)<\infty$ and $\mu_{C}(K, d ; \varrho)>0$, then $h_{\mathrm{esc}}(K, Q)>-\infty$.

\section{Proof.}

(i) Let $S \subset K(u, \tau)$ be a $(u, \tau, \varepsilon)$-separated set of maximal cardinality. Assume to the contrary that there is $y \in K(u, \tau)$ with $\varrho_{u, \tau}(x, y) \geq \varepsilon$ for all $x \in S$. Then also $S \cup\{y\}$ is $(u, \tau, \varepsilon)$-separated, in contradiction to the maximality of $S$. Hence, $S$ also $(u, \tau, \varepsilon)$-spans $K(u, \tau)$ implying that $r_{\text {span }}(u, \tau, \varepsilon, K, Q) \leq r_{\text {sep }}(u, \tau, \varepsilon, K, Q)$. Now, let $S \subset K(u, \tau)$ be any $(u, \tau, \varepsilon)$-separated set and $F \subset M$ any set which $(u, \tau, \varepsilon / 2)$-spans $K(u, \tau)$. Define a map $\alpha: S \rightarrow F$ by assigning to each $x \in S$ one $\alpha(x) \in F$ such that $\varrho_{u, \tau}(x, \alpha(x))<\varepsilon / 2$. Assume that $\alpha\left(x_{1}\right)=\alpha\left(x_{2}\right)$ for some $x_{1}, x_{2} \in S$. Then

$$
\varrho_{u, \tau}\left(x_{1}, x_{2}\right) \leq \varrho_{u, \tau}\left(x_{1}, \alpha\left(x_{1}\right)\right)+\varrho_{u, \tau}\left(\alpha\left(x_{2}\right), x_{2}\right)<\varepsilon .
$$

Hence, $x_{1}=x_{2}$, which shows that $\alpha$ is injective. Therefore, $\# S \leq \# F$ implying $r_{\text {sep }}(u, \tau, \varepsilon, K, Q) \leq r_{\text {span }}\left(u, \tau, \frac{\varepsilon}{2}, K, Q\right)$. By compactness of $K(u, \tau)$, it is clear that minimal $(u, \tau, \varepsilon)$-spanning sets are finite.

(ii) From the first statement it follows that

$$
\begin{aligned}
\varepsilon^{d} r_{\mathrm{span}}(u, \tau, \varepsilon, K, Q) & \leq \varepsilon^{d} r_{\mathrm{sep}}(u, \tau, \varepsilon, K, Q) \\
& \leq 2^{d}\left(\frac{\varepsilon}{2}\right)^{d} r_{\mathrm{span}}\left(u, \tau, \frac{\varepsilon}{2}, K, Q\right),
\end{aligned}
$$

which implies the assertion.

(iii) Let $\varrho$ and $\widetilde{\varrho}$ denote the distance functions induced by $g$ and $\widetilde{g}$, respectively. Then $\varrho$ is equivalent to $\widetilde{\varrho}$ on the compact set $Q$, which is shown as follows: For every $x \in M$ the norms induced by $g_{x}$ and $\widetilde{g}_{x}$ on $T_{x} M$ are equivalent, so in particular there is $L(x)>0$ such that $g_{x}(v, v)^{1 / 2} \leq L(x) \widetilde{g}_{x}(v, v)^{1 / 2}$ for all $v \in T_{x} M$. Since $g_{x}$ and $\widetilde{g}_{x}$ depend continuously on $x$, we can assume the same for $L(x)$. Now, let $x, y \in Q$ and let $\gamma:[0,1] \rightarrow M$ be a shortest geodesic from $x$ to $y$ with respect to $\widetilde{g}$, which exists by completeness. Then

$$
\begin{aligned}
\varrho(x, y) & \leq \int_{0}^{1} g_{\gamma(s)}(\dot{\gamma}(s), \dot{\gamma}(s))^{1 / 2} d s \\
& \leq \int_{0}^{1} L(\gamma(s)) \widetilde{g}_{\gamma(s)}(\dot{\gamma}(s), \dot{\gamma}(s))^{1 / 2} d s \\
& \leq \max _{s \in[0,1]} L(\gamma(s)) \int_{0}^{1} \widetilde{g}_{\gamma(s)}(\dot{\gamma}(s), \dot{\gamma}(s))^{1 / 2} d s \\
& =\max _{s \in[0,1]} L(\gamma(s)) \widetilde{\varrho}(x, y) .
\end{aligned}
$$


Let $A \subset M$ be the set defined as the union of the images of all shortest geodesics with respect to $\widetilde{g}$ joining points in $Q$. This set is obviously bounded, and hence for all $x, y \in Q$ we have

$$
\varrho(x, y) \leq L \widetilde{\varrho}(x, y) \text { with } L:=\max _{x \in \operatorname{cl} A} L(x) .
$$

Changing the roles of $g$ and $\widetilde{g}$ yields the claim. Now, let $S \subset K(u, \tau)$ be a maximal $(u, \tau, \varepsilon)$-separated set with respect to the metric $\varrho$. Then for all distinct $x, y \in S$ we have

$$
\begin{aligned}
\varepsilon & \leq \varrho_{u, \tau}(x, y)=\max _{t \in[0, \tau]} \varrho(\varphi(t, x, u), \varphi(t, y, u)) \\
& \leq L \max _{t \in[0, \tau]} \widetilde{\varrho}(\varphi(t, x, u), \varphi(t, y, u))=L \widetilde{\varrho}_{u, \tau}(x, y) .
\end{aligned}
$$

Hence, $S$ is $(u, \tau, \varepsilon / L)$-separated with respect to $\widetilde{\varrho}$ implying that

$$
L^{d}\left(\frac{\varepsilon}{L}\right)^{d} r_{\text {sep }}\left(u, \tau, \frac{\varepsilon}{L}, K, Q ; \widetilde{g}\right) \geq \varepsilon^{d} r_{\text {sep }}(u, \tau, \varepsilon, K, Q ; g),
$$

which yields $h_{\mathrm{esc}}(K, Q ; \widetilde{g}) \geq h_{\mathrm{esc}}(K, Q ; g)$. By changing the roles of $g$ and $\widetilde{g}$ the converse inequality follows.

(iv) Let $c, a>0$ be constants such that

$$
\varrho(\varphi(t, x, u), \varphi(t, y, u)) \leq c \mathrm{e}^{a t} \varrho(x, y)
$$

holds on a compact neighbourhood of $Q$, for $x, y \in Q$ with $\varrho(x, y)<\varepsilon$ for some sufficiently small $\varepsilon>0, t \geq 0$, and $u \in \mathcal{U}$ (cp. proof of [8, Theorem 12]). Then $\varrho(x, y)<\mathrm{e}^{-a \tau} \varepsilon$ implies

$$
\varrho(\varphi(t, x, u), \varphi(t, y, u)) \leq c \mathrm{e}^{a t} \varrho(x, y)<c \mathrm{e}^{a(t-\tau)} \varepsilon \leq c \varepsilon \text { for all } t \in[0, \tau] .
$$

For fixed $u \in \pi_{\mathcal{U}} \mathcal{K}_{Q}, \tau>0$, and $\varepsilon>0$ let $N(u, \tau, \varepsilon)$ be the minimal number of balls of radius $\mathrm{e}^{-a \tau} \varepsilon$ necessary to cover the compact set $K(u, \tau)$. We have shown that every such ball $B_{\mathrm{e}^{-a \tau} \varepsilon}(x)$ is contained in the Bowen-ball $B_{c \varepsilon}^{\tau}(u, x)$, which implies

$$
r_{\text {span }}(u, \tau, c \varepsilon, K, Q) \leq N(u, \tau, \varepsilon) .
$$

Hence, we obtain

$$
\begin{aligned}
\bar{r}_{\text {span }}(\tau, K, Q) & \leq \underset{\varepsilon \searrow 0}{\limsup _{u} \sup _{u}(c \varepsilon)^{d} N(u, \tau, \varepsilon)} \\
& =c^{d} \limsup _{\varepsilon \searrow 0} \sup _{u} \mathrm{e}^{a d \tau} \mu_{C}\left(K(u, \tau), d, \mathrm{e}^{-a \tau} \varepsilon\right) \\
& \leq c^{d} \mathrm{e}^{a d \tau} \limsup _{\varepsilon \searrow 0} \mu_{C}\left(K, d, \mathrm{e}^{-a \tau} \varepsilon\right)=c^{d} \mathrm{e}^{a d \tau} \mu_{C}(K, d) .
\end{aligned}
$$

This implies $h_{\mathrm{esc}}(K, Q) \leq a d<\infty$.

(v) It holds that $\varepsilon^{d} r_{\text {span }}(u, \tau, \varepsilon, K, Q)=\mu_{C}\left(K(u, \tau), d, \varepsilon ; \varrho_{u, \tau}\right)$ and hence

$$
\bar{r}_{\text {span }}(\tau, K, Q)=\limsup _{\varepsilon \searrow 0} \sup _{u \in \pi_{\mathcal{U}} \mathcal{K}_{Q}} \mu_{C}\left(K(u, \tau), d, \varepsilon ; \varrho_{u, \tau}\right) .
$$

Since $B_{\varepsilon}^{\tau}(u, x) \subset B_{\varepsilon}(x)$ for all $\varepsilon>0, \tau>0$, and $(u, x) \in \mathcal{U} \times M$, we have

$$
\mu_{C}\left(K(u, \tau), d, \varepsilon ; \varrho_{u, \tau}\right) \geq \mu_{C}(K(u, \tau), d, \varepsilon ; \varrho) .
$$


The assumption $h_{\text {inv }}^{*}(Q)<\infty$ guarantees finiteness of minimal $(\tau, K, Q)$-spanning sets (cp. [2, Rem. 3.2]). If $\mathcal{S}$ is such a set, then $K \subset \bigcup_{u \in \mathcal{S}} K(u, \tau)$ and hence

$$
\begin{aligned}
\mu_{C}(K, d, \varepsilon ; \varrho) & \leq \sum_{u \in \mathcal{S}} \mu_{C}(K(u, \tau), d, \varepsilon ; \varrho) \\
& \leq r_{\text {inv }}^{*}(\tau, K, Q) \sup _{u \in \pi_{\mathcal{U}} \mathcal{K}_{Q}} \mu_{C}(K(u, \tau), d, \varepsilon ; \varrho) .
\end{aligned}
$$

Altogether, we obtain

$$
\bar{r}_{\mathrm{span}}(\tau, K, Q) \geq \limsup _{\varepsilon \searrow 0} \frac{\mu_{C}(K, d, \varepsilon ; \varrho)}{r_{\mathrm{inv}}^{*}(\tau, K, Q)}=\frac{\mu_{C}(K, d ; \varrho)}{r_{\mathrm{inv}}^{*}(\tau, K, Q)} .
$$

Using (ii) and the assumptions, we can conclude that

$$
h_{\mathrm{esc}}(K, Q) \geq-h_{\mathrm{inv}}^{*}(K, Q) \geq-h_{\mathrm{inv}}^{*}(Q)>-\infty,
$$

which finishes the proof.

Remark 2.2 The quantity $h_{\mathrm{esc}}(K, Q)$ can be interpreted as a measure for the difference in the exponential growth rate of $\operatorname{vol}(K(u, \tau))$ and $\operatorname{vol}\left(B_{\varepsilon}^{\tau}(u, x)\right)$ for $\tau \rightarrow \infty$. Indeed, if $S$ is a maximal $(u, \tau, \varepsilon)$-separated subset of $K(u, \tau)$, then

$$
\operatorname{vol}(K(u, \tau)) \leq \sum_{x \in S} \operatorname{vol}\left(B_{\varepsilon}^{\tau}(u, x)\right) \leq r_{\text {sep }}(u, \tau, \varepsilon, K, Q) \sup _{x \in K(u, \tau)} \operatorname{vol}\left(B_{\varepsilon}^{\tau}(u, x)\right)
$$

implying that

$$
r_{\text {sep }}(u, \tau, \varepsilon, K, Q) \geq \frac{\operatorname{vol}(K(u, \tau))}{\sup _{x \in K(u, \tau)} \operatorname{vol}\left(B_{\varepsilon}^{\tau}(u, x)\right)} .
$$

On the other hand, the sets $B_{\varepsilon / 2}^{\tau}(u, x), x \in S$, are disjoint and contained in the $\varepsilon / 2$ neighbourhood of $K(u, \tau)$. Hence,

$$
\begin{aligned}
\operatorname{vol}\left(N_{\varepsilon / 2}(K(u, \tau))\right) & \geq \sum_{x \in S} \operatorname{vol}\left(B_{\varepsilon / 2}^{\tau}(u, x)\right) \\
& \geq r_{\text {sep }}(u, \tau, \varepsilon, K, Q) \inf _{x \in K(u, \tau)} \operatorname{vol}\left(B_{\varepsilon / 2}^{\tau}(u, x)\right),
\end{aligned}
$$

which implies

$$
r_{\mathrm{sep}}(u, \tau, \varepsilon, K, Q) \leq \frac{\operatorname{vol}\left(N_{\varepsilon / 2}(K(u, \tau))\right)}{\inf _{x \in K(u, \tau)} \operatorname{vol}\left(B_{\varepsilon / 2}^{\tau}(u, x)\right)}
$$

\subsection{Singular values and tensor analysis}

Let $\left(V,\langle\cdot, \cdot\rangle_{V}\right)$ and $\left(W,\langle\cdot, \cdot\rangle_{W}\right)$ be $d$-dimensional Euclidean spaces and $L: V \rightarrow W$ a linear mapping. Then we write

$$
\sigma_{1}(L) \geq \cdots \geq \sigma_{d}(L) \geq 0
$$


for the singular values of $L$, i.e., for the eigenvalues of the self-adjoint positive semidefinite operator $\sqrt{L^{*} L}: V \rightarrow V$. For $r \in\{0,1, \ldots, d\}$ we define the singular value function of $L$ of order $r$ by

$$
\omega_{r}(L):=\prod_{i=1}^{r} \sigma_{i}(L) .
$$

In particular, we will use the following basic properties of the singular values:

(i) If $L$ is an isomorphism between $d$-dimensional Euclidean spaces, then the singular values of $L$ are positive and $\sigma_{i}(L)^{-1}, i=1, \ldots, d$, are the singular values of $L^{-1}$.

(ii) For every linear mapping $L$ between $d$-dimensional Euclidean spaces it holds that $|\operatorname{det} L|=\omega_{d}(L)$.

Let $(V,\langle\cdot, \cdot\rangle)$ be a $d$-dimensional Euclidean space and $k \in\{1, \ldots, d\}$. Then $\bigwedge^{k} V$ denotes the $k$-th exterior power of $V$. A linear mapping $L: V \rightarrow V$ induces a linear mapping $L_{k}: \bigwedge^{k} V \rightarrow \bigwedge^{k} V$ by

$$
\begin{aligned}
L_{k}\left(v_{1} \wedge \ldots \wedge v_{k}\right):=L v_{1} \wedge \ldots \wedge v_{k}+v_{1} \wedge L v_{2} \wedge \ldots \wedge v_{k} & +\ldots \\
& +v_{1} \wedge \ldots \wedge L v_{k} .
\end{aligned}
$$

The Euclidean scalar product $\langle\cdot, \cdot\rangle$ on $V$ induces a scalar product on $\bigwedge^{k} V$ by

$$
\left\langle v_{1} \wedge \ldots \wedge v_{k}, w_{1} \wedge \ldots \wedge w_{k}\right\rangle_{\wedge^{k} V}:=\operatorname{det}\left(\left\langle v_{i}, w_{j}\right\rangle\right)_{i, j=1}^{k}
$$

with associated norm $\|\cdot\|_{\Lambda^{k} V}$. We will use the following lemma which can be found in [12, Lem. 1.2]:

Lemma 2.3 Let $(V,\langle\cdot, \cdot\rangle)$ be a d-dimensional Euclidean space and $L: V \rightarrow V$ a linear mapping. Then for all $k \in\{1, \ldots, d\}$ and all $v_{1}, \ldots, v_{k} \in V$ it holds that

$$
\left\langle L_{k}\left(v_{1} \wedge \ldots \wedge v_{k}\right), v_{1} \wedge \ldots \wedge v_{k}\right\rangle_{\wedge^{k} V}=\left\|v_{1} \wedge \ldots \wedge v_{k}\right\|_{\wedge^{k} V}^{2} \operatorname{tr}(L \circ Q),
$$

where $Q=Q\left(v_{1}, \ldots, v_{k}\right)$ denotes the orthogonal projection in $V$ onto the linear subspace spanned by $v_{1}, \ldots, v_{k}$.

Now, consider control system (2) and let $Q \subset M$ be a compact controlled invariant set. Let $E \rightarrow \mathcal{Q}, E=\bigcup_{(u, x) \in \mathcal{Q}}\{u\} \times E_{u, x}$, be a subbundle of (3) of rank $n, 0<n \leq d$. Then for $(u, x) \in \mathcal{Q}, \tau \in \mathbb{R}$, and $i=1, \ldots, n$ we define

$$
\sigma_{i}^{E}(u, x, \tau):=\sigma_{i}\left(\left.D \varphi_{\tau, u}(x)\right|_{E_{u, x}}\right) .
$$

For $r \in\{0,1, \ldots, n\}$ we write

$$
\omega_{r}^{E}(u, x, \tau):=\omega_{r}\left(\left.D \varphi_{\tau, u}(x)\right|_{E_{u, x}}\right) .
$$




\subsection{Ellipsoids in Euclidean space}

Let $\mathcal{E}$ be an ellipsoid in a $d$-dimensional Euclidean space $V$. Then the lengths of the half-axes of $\mathcal{E}$ are denoted by

$$
\sigma_{1}(\mathcal{E}) \geq \cdots \geq \sigma_{d}(\mathcal{E}) \geq 0 .
$$

Analogously to the definition of the singular value function, we set

$$
\omega_{r}(\mathcal{E}):=\prod_{i=1}^{r} \sigma_{i}(\mathcal{E})
$$

The following lemma on covering ellipsoids by balls can be found in [4] or [6, Lem. 4.2.2]:

Lemma 2.4 Let $\mathcal{E}$ be an ellipsoid in a d-dimensional Euclidean space $V$ and $\zeta>0$. Then $\mathcal{E}$ can be covered by $\left\lfloor 2^{r} \frac{\omega_{r}(\mathcal{E})}{\zeta^{r}}\right\rfloor$ balls of radii $\zeta \sqrt{r+1}$, where

$$
r= \begin{cases}0 & \text { for } \zeta>\sigma_{1}(\mathcal{E}) \\ l & \text { for } \sigma_{l+1}(\mathcal{E}) \leq \zeta \leq \sigma_{l}(\mathcal{E}), l \in\{1, \ldots, d-1\} \\ d & \text { for } \zeta \leq \sigma_{d}(\mathcal{E})\end{cases}
$$

\section{The Hausdorff measure of bowen-balls}

In this section, we generalize a result of Katrin Gelfert for estimating the outer Hausdorff measure of Bowen-balls (see [7, Lem. 1] or [6, Lem. 5.2.2]). Similar techniques are used in [5] for estimating the Hausdorff dimension of invariant sets of diffeomorphisms.

For the proof we will use another lemma which can be found in [6, Lem. 5.2.1]:

Lemma 3.1 Let $(M, g)$ be a Riemannian manifold, $U \subset M$ an open set, $\phi: U \rightarrow M$ a $C^{1}$-mapping and $Q \subset U$ a compact set with $\inf _{x \in Q}|\operatorname{det} D \phi(x)|>0$. Then there is $\varepsilon_{0}>0$ such that for all $x \in Q$ and $y \in B_{\varepsilon_{0}}(x)$ with $\phi(y) \in B_{\varepsilon_{0}}(\phi(x))$ the mapping $D \phi(y): T_{y} M \rightarrow T_{\phi(y)} M$ is invertible and the inequality

$$
\left\|\exp _{x}^{-1}(y)-D \phi(x)^{-1} \exp _{\phi(x)}^{-1}(\phi(y))\right\|_{T_{x} M} \leq \eta(x) \cdot \varepsilon_{0}
$$

holds with

$$
\eta(x):=\sup _{y: \phi(y) \in B_{\varepsilon_{0}}(\phi(x))}\left\|\tau_{y}^{x} \circ D \phi(y)^{-1} \circ \tau_{\phi(x)}^{\phi(y)}-D \phi(x)^{-1}\right\|,
$$

where $\tau_{y}^{x}$ is the isometric operator defined by parallel transport along the shortest geodesic from $y$ to $x$. Hence, for every $y \in B_{\varepsilon_{0}}(x)$ it holds that

$$
y=\exp _{x}\left(0_{x}+D \phi(x)^{-1} \exp _{\phi(x)}^{-1}(\phi(y))+w(y)\right)
$$

for a vector $w(y)$ with $\|w(y)\|_{T_{x} M} \leq \eta(x)$.

Now we can formulate and prove our main lemma. We like to emphasize that the proof is just a very slight modification of that given by Katrin Gelfert in [6] for the situation of a single (autonomous) dynamical system. 
Lemma 3.2 Consider control system (2) and let $Q \subset M$ be a compact controlled invariant set. Let $E$ be a subbundle of (3) of rank $n, 0<n \leq d$. Furthermore, let $u \in \pi_{\mathcal{U}} \mathcal{Q}$ and $\tau>0$ such that

$$
\inf _{x \in Q:(u, x) \in \mathcal{Q}} \omega_{n}^{E}(u, x, \tau)>1 .
$$

Then there is $\widetilde{\varepsilon}=\widetilde{\varepsilon}(u, \tau)>0$ such that for all $x \in Q$ with $(u, x) \in \mathcal{Q}$ and for all $\varepsilon \in(0, \widetilde{\varepsilon})$ it holds that

$$
\mu_{H}\left(B_{\varepsilon}^{\tau}(u, x), d, 8 \sqrt{d} \varepsilon\right) \leq\left(2^{4} \sqrt{d} \varepsilon\right)^{d} \omega_{n}^{E}(u, x, \tau)^{-1} .
$$

Proof. Let $E^{1}:=E$. If $n<d$, then we choose another subbundle $E^{0}$ of rank $d-n$, which is complementary to $E^{1}$, i.e., $E^{0} \oplus E^{1}=\bigcup_{(u, x) \in \mathcal{Q}}\{u\} \times T_{x} M$. If $n=d$, then $E^{1}=\bigcup_{(u, x) \in \mathcal{Q}}\{u\} \times T_{x} M$ and it is not necessary to consider another subbundle. The projections onto $E^{0}$ and $E^{1}$ are denoted by

$$
\pi_{i}: \bigcup_{(u, x) \in \mathcal{Q}}\{u\} \times T_{x} M \rightarrow E^{i}, \quad(u, v) \mapsto \pi_{i}(u) v, i=0,1 .
$$

We consider the map $\phi:=\varphi_{\tau, u}$. Since $\phi$ is smooth and $\inf _{x \in Q}|\operatorname{det} D \phi(x)|>0$ ( $\phi$ is a diffeomorphism), there are $\theta, \eta>0$ such that the inequalities

$$
\theta \geq\left(\inf _{x \in Q} \sigma_{d}(D \phi(x))\right)^{-1}, \quad \eta \leq\left(\sup _{x \in Q} \sigma_{1}(D \phi(x))\right)^{-1}
$$

hold. (Note that $|\operatorname{det} D \phi(x)|=\prod_{i=1}^{d} \sigma_{i}(D \phi(x))$.) Since

$$
\begin{aligned}
& \sup _{x \in Q} \sigma_{1}\left(D \phi(x)^{-1}\right)=\sup _{x \in Q} \frac{1}{\sigma_{d}(D \phi(x))}, \\
& \inf _{x \in Q} \sigma_{d}\left(D \phi(x)^{-1}\right)=\inf _{x \in Q} \frac{1}{\sigma_{1}(D \phi(x))},
\end{aligned}
$$

we have

$$
\sup _{x \in Q} \sigma_{1}\left(D \phi(x)^{-1}\right) \leq \theta \text { and } \eta \leq \inf _{x \in Q} \sigma_{d}\left(D \phi(x)^{-1}\right) .
$$

From the assumptions of the lemma and elementary transformations we obtain

$$
\begin{aligned}
\inf _{x \in Q:(u, x) \in \mathcal{Q}} \omega_{n}^{E^{1}}(u, x, \tau)>1 \\
\Leftrightarrow \sup _{x \in Q:(u, x) \in \mathcal{Q}} \omega_{n}\left(\left.D \phi(x)^{-1}\right|_{D \phi(x) E_{u, x}^{1}}\right)<1
\end{aligned}
$$

and therefore

$$
\begin{aligned}
\inf _{x \in Q:(u, x) \in \mathcal{Q}} \sigma_{1}^{E^{1}}(u, x, \tau)>1 \\
\quad \Leftrightarrow \quad \sup _{x \in Q:(u, x) \in \mathcal{Q}} \sigma_{n}\left(\left.D \phi(x)^{-1}\right|_{D \phi(x) E_{u, x}^{1}}\right)<1 .
\end{aligned}
$$

(Note that $\omega_{n}^{E^{1}}(u, x, \tau)=\sigma_{1}^{E^{1}}(u, x, \tau) \cdot \ldots \cdot \sigma_{n}^{E^{1}}(u, x, \tau)$ and $\sigma_{1}(\cdot)$ is the greatest singular value.) We choose $\widetilde{\varepsilon}>0$ small enough such that the following conditions hold:

(i) $\widetilde{\varepsilon}$ is smaller than $\varepsilon_{0}$ from Lemma 3.1 applied to $\phi$ and the compact set $Q$. 
(ii) For any $x \in Q$ we have

$$
\left\|\tau_{y}^{x} \circ D \phi(y)^{-1} \circ \tau_{\phi(x)}^{\phi(y)}-D \phi(x)^{-1}\right\| \leq \eta
$$

for all $y \in B_{\widetilde{\varepsilon}}(x)$ with $\phi(y) \in B_{\widetilde{\varepsilon}}(\phi(x))$.

(iii) The inequality

$$
\varrho\left(\exp _{y}\left(v_{1}\right), \exp _{y}\left(v_{2}\right)\right) \leq 2\left\|v_{1}-v_{2}\right\|_{T_{y} M}
$$

holds for all $y \in Q$ and $v_{1}, v_{2} \in B_{\widetilde{\varepsilon}}\left(0_{y}\right)$ with $\left\|v_{1}-v_{2}\right\|_{T_{y} M} \leq(\theta+\eta)(2 \sqrt{d}+1) \widetilde{\varepsilon}$.

Now we fix a point $x \in Q$ with $(u, x) \in \mathcal{Q}$ and a number $\varepsilon \in(0, \widetilde{\varepsilon})$. Obviously, we have $B_{\varepsilon}^{\tau}(u, x) \subset B_{\varepsilon}(x)$. By applying Lemma 3.1 to $\phi=\varphi_{\tau, u}$, with (11) we get

$$
\exp _{x}^{-1}\left(B_{\varepsilon}^{\tau}(u, x)\right) \subset D \phi(x)^{-1} B_{\varepsilon}\left(0_{\phi(x)}\right)+B_{\eta \varepsilon}\left(0_{x}\right) .
$$

With the chosen splitting of $\bigcup_{(u, x) \in \mathcal{Q}}\{u\} \times T_{x} M$ we obtain

$$
\exp _{x}^{-1}\left(B_{\varepsilon}^{\tau}(u, x)\right) \subset \pi_{1}(u) D \phi(x)^{-1} B_{\varepsilon}\left(0_{\phi(x)}\right) \oplus \pi_{0}(u) B_{\varepsilon}\left(0_{x}\right)+B_{\eta \varepsilon}\left(0_{x}\right) .
$$

The set $\mathcal{E}_{1}:=\left(\pi_{1}(u) \circ D \phi(x)^{-1}\right) B_{\varepsilon}\left(0_{\phi(x)}\right)$ is an ellipsoid in $E_{u, x}^{1}$ with half-axes of lengths

$$
\sigma_{i}\left(\mathcal{E}_{1}\right)=\sigma_{i}\left(\left.D \phi(x)^{-1}\right|_{D \phi(x) E_{u, x}^{1}}\right) \varepsilon, \quad i=1, \ldots, n .
$$

The set $\mathcal{E}_{0}:=\pi_{0}(u)\left(\exp _{x}^{-1}\left(B_{\varepsilon}(x)\right)\right)$ is an $\varepsilon$-ball in $E_{u, x}^{0}$ and hence an ellipsoid with halfaxes of lengths $\sigma_{1}\left(\mathcal{E}_{0}\right)=\cdots=\sigma_{d-n}\left(\mathcal{E}_{0}\right)=\varepsilon$. The set $\mathcal{E}_{1}+B_{\eta \varepsilon}\left(0_{x}\right)$ is obviously contained in an ellipsoid $\mathcal{E}_{1}^{\prime}$ with half-axes of lengths

$$
\sigma_{i}\left(\mathcal{E}_{1}^{\prime}\right)=\left(1+\frac{\eta \varepsilon}{\sigma_{n}\left(\mathcal{E}_{1}\right)}\right) \sigma_{i}\left(\mathcal{E}_{1}\right), \quad i=1, \ldots, n .
$$

From (10) and (15) we conclude that

$$
\sigma_{n}\left(\mathcal{E}_{1}\right)<\varepsilon .
$$

With $\varepsilon+\eta \varepsilon=\left(1+\frac{\eta \varepsilon}{\varepsilon}\right) \varepsilon$ it follows that $\mathcal{E}_{0}+B_{\eta \varepsilon}\left(0_{x}\right)$ is contained in an ellipsoid $\mathcal{E}_{0}^{\prime} \subset E_{u, x}^{0}$ with half-axes of lengths

$$
\sigma_{i}\left(\mathcal{E}_{0}^{\prime}\right)=\left(1+\frac{\eta \varepsilon}{\sigma_{n}\left(\mathcal{E}_{1}\right)}\right) \varepsilon, \quad i=1, \ldots, d-n .
$$

We set

$$
\zeta:=\left(1+\frac{\eta \varepsilon}{\sigma_{n}\left(\mathcal{E}_{1}\right)}\right) \sigma_{n}\left(\mathcal{E}_{1}\right) .
$$

By Lemma 2.4 we can cover $\mathcal{E}_{1}^{\prime}$ with $N_{1}:=\left\lfloor 2^{n} \frac{\omega_{n}\left(\mathcal{E}_{1}^{\prime}\right)}{\zeta^{n}}\right\rfloor$ balls of radii $\zeta \sqrt{n+1}$ and $\mathcal{E}_{0}^{\prime}$ with

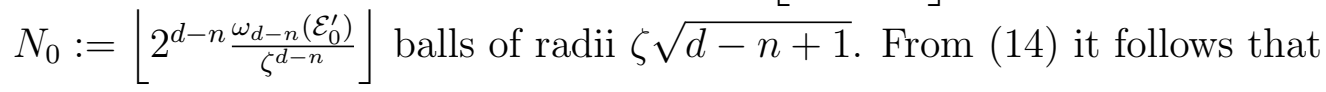

$$
\exp _{x}^{-1}\left(B_{\varepsilon}^{\tau}(u, x)\right) \subset \mathcal{E}_{1} \oplus \mathcal{E}_{0}+B_{\eta \varepsilon}\left(0_{x}\right) \subset \mathcal{E}_{1}^{\prime} \oplus \mathcal{E}_{0}^{\prime},
$$

and hence the set $\exp _{x}^{-1}\left(B_{\varepsilon}^{\tau}(u, x)\right)$ can be covered with $N_{1} N_{0}$ balls of radii $2 \zeta \sqrt{d}$. (The product of a Euclidean ball of radius $\zeta \sqrt{n+1}$ and one of radius $\zeta \sqrt{d-n+1}$ is contained in a ball of radius $\left((\zeta \sqrt{n+1})^{2}+(\zeta \sqrt{d-n+1})^{2}\right)^{1 / 2}=\zeta \sqrt{d+2} \leq 2 \zeta \sqrt{d}$. $)$ From $(9)$ and (13) it follows that each of these balls lies in the ball of radius $(\theta+\eta)(2 \sqrt{d}+1) \varepsilon$ and 
centre $0_{x} \in T_{x} M$. This is shown as follows: By (13) each $v \in \exp _{x}^{-1}\left(B_{\varepsilon}^{\tau}(u, x)\right)$ can be written as $v=w_{1}+w_{2}$ with $\left\|w_{1}\right\| \leq \sigma_{1}\left(D \phi(x)^{-1}\right) \varepsilon,\left\|w_{2}\right\| \leq \eta \varepsilon$. Hence, $\|v\| \leq \varepsilon(\theta+\eta)$. Now consider a ball with radius $2 \zeta \sqrt{d}$ around $v$. Since $2 \zeta \sqrt{d}=2 \sqrt{d}\left(\sigma_{n}\left(\mathcal{E}_{1}\right)+\eta \varepsilon\right) \leq$ $2 \sqrt{d} \varepsilon\left(\sigma_{1}\left(D \phi(x)^{-1}\right)+\eta\right) \leq 2 \sqrt{d} \varepsilon(\theta+\eta)$, we obtain that the norm of such a vector is bounded by $\varepsilon(\theta+\eta)+2 \sqrt{d} \varepsilon(\theta+\eta)=(\theta+\eta)(2 \sqrt{d}+1) \varepsilon$. If one maps this cover with $\exp _{x}$ down to the manifold, then, by $(12), B_{\varepsilon}^{\tau}(u, x)$ is covered by $N_{1} N_{0}$ balls of radii $4 \zeta \sqrt{d}$. Hence, we obtain

$$
\mu_{H}\left(B_{\varepsilon}^{\tau}(u, x), d, 4 \zeta \sqrt{d}\right) \leq N_{1} N_{0}(4 \zeta \sqrt{d})^{d} .
$$

This implies

$$
\begin{aligned}
\mu_{H} & \left(B_{\varepsilon}^{\tau}(u, x), d, 4 \zeta \sqrt{d}\right) \leq \frac{2^{n} \omega_{n}\left(\mathcal{E}_{1}^{\prime}\right)}{\zeta^{n}} \frac{2^{d-n} \omega_{d-n}\left(\mathcal{E}_{0}^{\prime}\right)}{\zeta^{d-n}}(4 \zeta \sqrt{d})^{d} \\
& \leq 2^{d} \frac{\left(1+\frac{\eta \varepsilon}{\sigma_{n}\left(\mathcal{E}_{1}\right)}\right)^{d} \omega_{n}\left(\mathcal{E}_{1}\right) \omega_{d-n}\left(\mathcal{E}_{0}\right)}{\left(1+\frac{\eta \varepsilon}{\sigma_{n}\left(\mathcal{E}_{1}\right)}\right)^{d} \sigma_{n}\left(\mathcal{E}_{1}\right)^{d}}(4 \zeta \sqrt{d})^{d} \\
& =\left(2^{3} \sqrt{d}\right)^{d} \omega_{n}\left(\left.D \phi(x)^{-1}\right|_{D \phi(x) E_{u, x}^{1}}\right)\left(\frac{\zeta}{\sigma_{n}\left(\left.D \phi(x)^{-1}\right|_{D \phi(x) E_{u, x}^{1}}\right)}\right)^{d} \\
& =\left(2^{3} \sqrt{d} \varepsilon\right)^{d} \omega_{n}^{E^{1}}(u, x, \tau)^{-1}\left(\frac{\sigma_{n}\left(\left.D \phi(x)^{-1}\right|_{D \phi(x) E_{u, x}^{1}}\right)+\eta}{\sigma_{n}\left(\left.D \phi(x)^{-1}\right|_{D \phi(x) E_{u, x}^{1}}\right)}\right)^{d} .
\end{aligned}
$$

By choice of $\eta$ we have

$$
\eta \leq \inf _{y \in Q} \sigma_{d}\left(D \phi(y)^{-1}\right) \leq \sigma_{d}\left(D \phi(x)^{-1}\right) \leq \sigma_{n}\left(\left.D \phi(x)^{-1}\right|_{D \phi(x) E_{u, x}^{1}}\right) .
$$

Hence,

$$
\mu_{H}\left(B_{\varepsilon}^{\tau}(u, x), d, 4 \zeta \sqrt{d}\right) \leq\left(2^{4} \sqrt{d} \varepsilon\right)^{d} \omega_{n}^{E^{1}}(u, x, \tau)^{-1} .
$$

We can assume that $\eta \leq 1$ and hence, with (10), we have

$$
\zeta=\varepsilon\left(\sigma_{n}\left(\left.D \phi(x)^{-1}\right|_{D \phi(x) E_{u, x}^{1}}\right)+\eta\right)<2 \varepsilon .
$$

This gives

$$
\mu_{H}\left(B_{\varepsilon}^{\tau}(u, x), d, 8 \sqrt{d} \varepsilon\right) \leq \mu_{H}\left(B_{\varepsilon}^{\tau}(u, x), d, 4 \zeta \sqrt{d}\right),
$$

which implies the result.

\section{The main result}

Now we are in position to prove our main theorem:

Theorem 4.1 Consider control system (2) and let $Q \subset M$ be a compact controlled invariant set with $h_{\mathrm{inv}}^{*}(Q)<\infty$. Let $K \subset Q$ be a compact set of positive d-dimensional 
Lower bounds for the strict invariance entropy

Hausdorff measure and let $E$ be a subbundle of (3) of rank $n$. Assume that there is $\tau_{0}>0$ such that for all $u \in \pi_{\mathcal{U}} \mathcal{Q}$ and $\tau \geq \tau_{0}$

$$
\inf _{x \in Q:(u, x) \in \mathcal{Q}} \omega_{n}^{E}(u, x, \tau)>1 .
$$

Then it holds that

$$
h_{\mathrm{inv}}^{*}(K, Q) \geq \limsup _{\tau \rightarrow \infty} \frac{1}{\tau} \inf _{(u, x) \in \mathcal{K}_{Q}} \ln \omega_{n}^{E}(u, x, \tau)-h_{\mathrm{esc}}(K, Q) .
$$

Proof. Fix an arbitrary $\tau \geq \tau_{0}$ and a minimal $(\tau, K, Q)$-spanning set $\mathcal{S}^{*}$. Since $h_{\text {inv }}^{*}(Q)<\infty$ by assumption, $\mathcal{S}^{*}$ is finite (cp. [2, Rem. 3.2]). Moreover, we can assume that $\mathcal{S}^{*} \subset \pi_{\mathcal{U}} \mathcal{K}_{Q} \subset \pi_{\mathcal{U}} \mathcal{Q}$. For each $u \in \mathcal{S}^{*}$ Lemma 3.2 yields an $\widetilde{\varepsilon}(u)>0$ such that for all $x \in Q$ with $(u, x) \in \mathcal{Q}$ and $\varepsilon \in(0, \widetilde{\varepsilon}(u))$ the estimate

$$
\mu_{H}\left(B_{\varepsilon}^{\tau}(u, x), d, 8 \sqrt{d \varepsilon}\right) \leq\left(2^{4} \sqrt{d} \varepsilon\right)^{d} \omega_{n}^{E}(u, x, \tau)^{-1}
$$

holds. Let $\widetilde{\varepsilon}:=\min _{u \in \mathcal{S}^{*}} \widetilde{\varepsilon}(u)$. For each $\varepsilon \in(0, \widetilde{\varepsilon})$ and $u \in \mathcal{S}^{*}$ let $S_{u, \tau, \varepsilon}$ be a maximal $(u, \tau, \varepsilon)$-separated subset of $K(u, \tau)$. Then, since $K=\bigcup_{u \in \mathcal{S}^{*}} K(u, \tau)$ and $K(u, \tau) \subset \bigcup_{x \in S_{u, \tau, \varepsilon}} B_{\varepsilon}^{\tau}(u, x)$, for each $\varepsilon \in(0, \widetilde{\varepsilon})$ we obtain

$$
\begin{aligned}
\mu_{H}(K, d, 8 \sqrt{d} \varepsilon) & \leq \sum_{u \in \mathcal{S}^{*}} \mu_{H}(K(u, \tau), d, 8 \sqrt{d} \varepsilon) \\
& \leq r_{\mathrm{inv}}^{*}(\tau, K, Q) \max _{u \in \mathcal{S}^{*}} \mu_{H}(K(u, \tau), d, 8 \sqrt{d} \varepsilon) \\
& \leq r_{\mathrm{inv}}^{*}(\tau, K, Q) \max _{u \in \mathcal{S}^{*}} \sum_{x \in S_{u, \tau, \varepsilon}} \mu_{H}\left(B_{\varepsilon}^{\tau}(u, x), d, 8 \sqrt{d} \varepsilon\right) \\
& \leq r_{\mathrm{inv}}^{*}(\tau, K, Q) \max _{u \in \mathcal{S}^{*}} \sum_{x \in S_{u, \tau, \varepsilon}}\left(2^{4} \sqrt{d} \varepsilon\right)^{d} \omega_{n}^{E}(u, x, \tau)^{-1}
\end{aligned}
$$

and

$$
\begin{aligned}
\max _{u \in \mathcal{S}^{*}} \sum_{x \in S_{u, \tau, \varepsilon}} \varepsilon^{d} \omega_{n}^{E}(u, x, \tau)^{-1} & \leq \max _{u \in \mathcal{S}^{*}}\left(\varepsilon^{d} r_{\operatorname{sep}}(u, \tau, \varepsilon, K, Q) \sup _{x \in K(u, \tau)} \omega_{n}^{E}(u, x, \tau)^{-1}\right) \\
& \leq\left(\sup _{u \in \pi_{\mathcal{U}} \mathcal{K}_{Q}} \varepsilon^{d} r_{\mathrm{sep}}(u, \tau, \varepsilon, K, Q)\right)\left(\sup _{(u, x) \in \mathcal{K}_{Q}} \omega_{n}^{E}(u, x, \tau)^{-1}\right) .
\end{aligned}
$$

With $\gamma:=\left(2^{4} \sqrt{d}\right)^{-d}$ this implies the estimate

$$
r_{\text {inv }}^{*}(\tau, K, Q) \geq \gamma \mu_{H}(K, d, 8 \sqrt{d} \varepsilon) \bar{r}_{\text {sep }}(\tau, \varepsilon, K, Q)^{-1} \inf _{(u, x) \in \mathcal{K}_{Q}} \omega_{n}^{E}(u, x, \tau) .
$$

Applying the logarithm to this inequality yields

$$
\begin{aligned}
\ln r_{\text {inv }}^{*}(\tau, K, Q) & \geq \ln \left(\gamma \mu_{H}(K, d, 8 \sqrt{d} \varepsilon)\right) \\
& -\ln \bar{r}_{\mathrm{sep}}(\tau, \varepsilon, K, Q)+\inf _{(u, x) \in \mathcal{K}_{Q}} \ln \omega_{n}^{E}(u, x, \tau) .
\end{aligned}
$$


Lower bounds for the strict invariance entropy

Since this holds for all $\varepsilon \in(0, \widetilde{\varepsilon})$, we also get

$$
\begin{aligned}
\ln r_{\text {inv }}^{*}(\tau, K, Q) & \geq \lim _{\varepsilon \searrow 0} \ln \left(\gamma \mu_{H}(K, d, 8 \sqrt{d} \varepsilon)\right) \\
& -\limsup _{\varepsilon \searrow 0} \ln \bar{r}_{\text {sep }}(\tau, \varepsilon, K, Q)+\inf _{(u, x) \in \mathcal{K}_{Q}} \ln \omega_{n}^{E}(u, x, \tau) \\
& =\ln \left(\gamma \mu_{H}(K, d)\right)-\ln \bar{r}_{\mathrm{sep}}(\tau, K, Q)+\inf _{(u, x) \in \mathcal{K}_{Q}} \ln \omega_{n}^{E}(u, x, \tau) .
\end{aligned}
$$

Dividing by $\tau$ and letting $\tau$ go to infinity yields

$$
\begin{aligned}
h_{\text {inv }}^{*}(K, Q) & \geq \limsup _{\tau \rightarrow \infty}\left[-\frac{1}{\tau} \ln \bar{r}_{\text {sep }}(\tau, K, Q)+\frac{1}{\tau} \inf _{(u, x) \in \mathcal{K}_{Q}} \ln \omega_{n}^{E}(u, x, \tau)\right] \\
& \geq \limsup _{\tau \rightarrow \infty} \frac{1}{\tau} \inf _{(u, x) \in \mathcal{K}_{Q}} \ln \omega_{n}^{E}(u, x, \tau)-\limsup _{\tau \rightarrow \infty} \frac{1}{\tau} \ln \bar{r}_{\text {sep }}(\tau, K, Q) .
\end{aligned}
$$

This finishes the proof.

Corollary 4.2 Assume that the subbundle $E$ in Theorem 4.1 is invariant under the cocycle $\varphi$, i.e.,

$$
D \varphi_{t, u} E_{u, x}=E_{\Theta_{t} u, \varphi_{t, u}(x)} \text { for all } t \geq 0, \quad(u, x) \in \mathcal{Q} .
$$

Then

$h_{\text {inv }}^{*}(K, Q) \geq \limsup _{\tau \rightarrow \infty} \frac{1}{\tau} \inf _{(u, x) \in \mathcal{K}_{Q}} \int_{0}^{\tau} \operatorname{tr}\left[\nabla F_{u(s)}\left(\varphi_{s, u}(x)\right) \circ Q\left(\Theta_{s} u, \varphi_{s, u}(x)\right)\right] d s-h_{\mathrm{esc}}(K, Q)$, where $Q(u, x): T_{x} M \rightarrow E_{u, x}$ is the orthogonal projection.

Proof. We have

$$
\omega_{n}^{E}(u, x, \tau)=\omega_{n}\left(\left.D \varphi_{\tau, u}(x)\right|_{E_{u, x}}\right)=\left|\operatorname{det} D \varphi_{\tau, u}(x)\right|_{E_{u, x}} \mid .
$$

We fix $(u, x) \in \mathcal{K}_{Q}$ and write

$$
L(\tau):=\left.D \varphi_{\tau, u}(x)\right|_{E_{u, x}}: E_{u, x} \rightarrow E_{\Theta_{\tau} u, \varphi_{\tau, u}(x)}, \quad \tau \geq 0 .
$$

Let $\left(v_{1}, \ldots, v_{n}\right)$ be an orthonormal basis of $E_{u, x}$. Then

$$
\begin{aligned}
(\operatorname{det} L(\tau))^{2} & =\operatorname{det}\left(L(\tau)^{*} L(\tau)\right)=\operatorname{det}\left(\left\langle L(\tau)^{*} L(\tau) v_{i}, v_{j}\right\rangle\right)_{i, j=1}^{n} \\
& =\operatorname{det}\left(\left\langle L(\tau) v_{i}, L(\tau) v_{j}\right\rangle\right)_{i, j=1}^{n} .
\end{aligned}
$$

Using that $v_{i}(\tau):=L(\tau) v_{i}$ solves the Riemannian variational equation for each $i \in$ $\{1, \ldots, n\}$ (cp. [8, Prop. 9]), we obtain for almost all $\tau \geq 0$ that

$$
\begin{aligned}
\frac{1}{2} \frac{\mathrm{d}}{\mathrm{d} \tau}(\operatorname{det} L(\tau))^{2} & =\frac{1}{2} \frac{\mathrm{d}}{\mathrm{d} \tau}\left\langle v_{1}(\tau) \wedge \ldots \wedge v_{n}(\tau), v_{1}(\tau) \wedge \ldots \wedge v_{n}(\tau)\right\rangle_{\wedge^{n} T_{\varphi(\tau, x, u)} M} \\
& =\left\langle\frac{\mathrm{D} v_{1}}{\mathrm{~d} \tau}(\tau) \wedge \ldots \wedge v_{n}(\tau), v_{1}(\tau) \wedge \ldots \wedge v_{n}(\tau)\right\rangle_{\wedge^{n} T_{\varphi(\tau, x, u)} M} \\
& +\ldots+ \\
& \left\langle v_{1}(\tau) \wedge \ldots \wedge \frac{\mathrm{D} v_{n}}{\mathrm{~d} \tau}(\tau), v_{1}(\tau) \wedge \ldots \wedge v_{n}(\tau)\right\rangle_{\wedge^{n} T_{\varphi(\tau, x, u)} M} \\
& =\left\langle\nabla F_{u(\tau)}\left(\varphi_{\tau, u}(x)\right) v_{1}(\tau) \wedge \ldots \wedge v_{n}(\tau), v_{1}(\tau) \wedge \ldots \wedge v_{n}(\tau)\right\rangle_{\wedge^{n} T_{\varphi(\tau, x, u)} M} \\
& +\ldots+ \\
& \left\langle v_{1}(\tau) \wedge \ldots \wedge \nabla F_{u(\tau)}\left(\varphi_{\tau, u}(x)\right) v_{n}(\tau), v_{1}(\tau) \wedge \ldots \wedge v_{n}(\tau)\right\rangle_{\wedge^{n} T_{\varphi(\tau, x, u)} M} .
\end{aligned}
$$


With Lemma 2.3 and assumption (20) we thus obtain that $\operatorname{det} L(\tau)$ satisfies the scalar linear differential equation

$$
\begin{aligned}
\frac{\mathrm{d}}{\mathrm{d} \tau} \operatorname{det} L(\tau) & =\frac{\frac{\mathrm{d}}{\mathrm{d} \tau}(\operatorname{det} L(\tau))^{2}}{2 \operatorname{det} L(\tau)} \\
& =\operatorname{tr}\left[\nabla F_{u(\tau)}\left(\varphi_{\tau, u}(x)\right) \circ Q\left(\Theta_{\tau} u, \varphi_{\tau, u}(x)\right)\right] \operatorname{det} L(\tau) .
\end{aligned}
$$

This gives

$$
\begin{aligned}
|\operatorname{det} L(\tau)| & =\operatorname{det} L(\tau) \\
& =\exp \left(\int_{0}^{\tau} \operatorname{tr}\left[\nabla F_{u(s)}\left(\varphi_{s, u}(x)\right) \circ Q\left(\Theta_{s} u, \varphi_{s, u}(x)\right)\right] d s\right),
\end{aligned}
$$

since $\operatorname{det} L(0)=\operatorname{det} \operatorname{id}_{E_{u, x}}=1$.

\section{Uniformly expanding systems}

We call the control system (2) uniformly expanding on a set $A \subset M$ with respect to the Riemannian metric $g$ if there are constants $C, \lambda>0$ such that for all $u \in \mathcal{U}, x_{1}, x_{2} \in A$, and $\tau>0$ with $\varphi\left([0, \tau], x_{i}, u\right) \subset A(i=1,2)$ it holds that

$$
\varrho\left(\varphi\left(\tau, x_{1}, u\right), \varphi\left(\tau, x_{2}, u\right)\right) \geq C \mathrm{e}^{\lambda \tau} \varrho\left(x_{1}, x_{2}\right) .
$$

By [9, Prop. 3.2.8] a sufficient condition for uniform expansiveness is the existence of a constant $\beta>0$ such that

$$
\lambda_{\min }\left(S \nabla F_{u}(x)\right) \geq \beta
$$

for all $(x, u)$ in a sufficiently large superset of $Q \times U$. Here $S \nabla F_{u}(x)$ denotes the symmetrized covariant derivative of the vector field $F_{u}$ at $x$ and $\lambda_{\min }(\cdot)$ is the minimal eigenvalue.

Proposition 5.1 If system (2) is uniformly expanding on the compact controlled invariant set $Q$, then $h_{\mathrm{esc}}(K, Q) \leq 0$ for every compact set $K \subset Q$.

Proof. For arbitrary $u \in \pi_{\mathcal{U}} \mathcal{K}_{Q}, \tau>0$, and $\varepsilon>0$ let $S$ be a $(u, \tau, \varepsilon)$-separated subset of $K(u, \tau)$. Choose distinct points $x_{1}, x_{2} \in S$ and let $s=s\left(x_{1}, x_{2}\right) \in[0, \tau]$ such that $\varrho\left(\varphi\left(s, x_{1}, u\right), \varphi\left(s, x_{2}, u\right)\right)=\varrho_{u, \tau}\left(x_{1}, x_{2}\right)$. Then, by the cocycle property, it holds that

$$
\begin{aligned}
\varrho\left(\varphi\left(\tau, x_{1}, u\right), \varphi\left(\tau, x_{2}, u\right)\right) & =\varrho\left(\varphi\left(\tau-s, \varphi\left(s, x_{1}, u\right), \Theta_{s} u\right), \varphi\left(\tau-s, \varphi\left(s, x_{2}, u\right), \Theta_{s} u\right)\right) \\
& \geq C \mathrm{e}^{\lambda(\tau-s)} \varrho\left(\varphi\left(s, x_{1}, u\right), \varphi\left(s, x_{2}, u\right)\right) \\
& \geq C \mathrm{e}^{\lambda(\tau-s)} \varepsilon \geq C \varepsilon .
\end{aligned}
$$

Hence, $\varphi_{\tau, u}(S)$ is a $C \varepsilon$-separated subset of $Q$, which has the same cardinality as $S$ (since $\varphi_{\tau, u}$ is injective). By compactness we can cover $Q$ with finitely many balls $B_{\delta}\left(x_{1}\right), \ldots, B_{\delta}\left(x_{n}\right)$ of a fixed radius $\delta>0$ such that $\exp _{x_{i}}^{-1}\left(B_{\delta}\left(x_{i}\right)\right)=B_{\delta}\left(0_{x_{i}}\right)$ and

$$
\varrho\left(\exp _{x_{i}}(v), \exp _{x_{i}}(w)\right) \leq 2\|v-w\| \text { for all } v, w \in B_{\delta}\left(0_{x_{i}}\right)
$$


for $i=1, \ldots, n$. The latter is possible since $D \exp _{x}\left(0_{x}\right)=\mathrm{id}_{T_{x} M}$ for all $x \in M$. Then we have

$$
\begin{aligned}
\# \varphi_{\tau, u}(S) \leq \sum_{i=1}^{n} & \#\left(\varphi_{\tau, u}(S) \cap B_{\delta}\left(x_{i}\right)\right) \\
& \leq n \max _{i=1}^{n} \underbrace{\# \exp _{x_{i}}^{-1}\left(\varphi_{\tau, u}(S) \cap B_{\delta}\left(x_{i}\right)\right)}_{=: N_{i}} .
\end{aligned}
$$

By (22), the set $\exp _{x_{i}}^{-1}\left(\varphi_{\tau, u}(S) \cap B_{\delta}\left(x_{i}\right)\right)$ is a $(C \varepsilon) / 2$-separated subset of $B_{\delta}\left(0_{x_{i}}\right)$. Hence, $B_{\delta+(C \varepsilon) / 4}\left(0_{x_{i}}\right)$ contains $N_{i}$ disjoint balls of radii $(C \varepsilon) / 4$. This implies

$$
\left(\delta+\frac{C \varepsilon}{4}\right)^{d} \geq N_{i}\left(\frac{C \varepsilon}{4}\right)^{d} \Rightarrow N_{i} \leq\left(\frac{4 \delta+C \varepsilon}{C \varepsilon}\right)^{d} .
$$

Hence, we get

$$
\# S=\# \varphi_{\tau, u}(S) \leq n\left(\frac{4 \delta+C \varepsilon}{C \varepsilon}\right)^{d} .
$$

Therefore, we obtain

$$
\begin{aligned}
\bar{r}_{\text {sep }}(\tau, K, Q) & =\underset{\varepsilon \searrow 0}{\limsup } \bar{r}_{\text {sep }}(\tau, \varepsilon, K, Q) \\
& \leq \limsup _{\varepsilon \searrow 0} n\left(\frac{4 \delta+C \varepsilon}{C}\right)^{d}=n\left(\frac{4 \delta}{C}\right)^{d},
\end{aligned}
$$

which implies the assertion.

Corollary 5.2 Assume that control system (2) is uniformly expanding on a compact controlled invariant set $Q$ with $h_{\mathrm{inv}}^{*}(Q)<\infty$. Then for every compact set $K \subset Q$ of positive volume we have

$$
h_{\mathrm{inv}}^{*}(K, Q) \geq \limsup _{\tau \rightarrow \infty} \frac{1}{\tau} \inf _{(u, x) \in \mathcal{K}_{Q}} \int_{0}^{\tau} \operatorname{div} F_{u(s)}\left(\varphi_{s, u}(x)\right) d s .
$$

In particular, this implies $h_{\mathrm{inv}}^{*}(K, Q)>0$.

Proof. Combine Corollary 4.2 with $E=\bigcup_{(u, x) \in \mathcal{Q}}\{u\} \times T_{x} M$ and Proposition 5.1. In order to show that assumption (17) of Corollary 4.2 holds, note that (21) implies

$$
\left\|D \varphi_{\tau, u}(x) v\right\| \geq C \mathrm{e}^{\lambda \tau}\|v\|
$$

for all $\tau \geq 0, x \in Q, u \in \mathcal{U}$, and $v \in T_{x} M$, since for $x_{1}, x_{2} \in Q$ with small distance $\delta=\varrho\left(x_{1}, x_{2}\right)$ we can choose a shortest geodesic $\gamma:[0, \delta] \rightarrow M$ from $x_{1}$ to $x_{2}$ and with $v:=\dot{\gamma}(0)$ we obtain

$$
\begin{aligned}
C \mathrm{e}^{\lambda \tau} \delta & =C \mathrm{e}^{\lambda \tau} \varrho\left(x_{1}, x_{2}\right) \leq \varrho\left(\varphi\left(\tau, x_{1}, u\right), \varphi\left(\tau, x_{2}, u\right)\right) \\
& \leq \int_{0}^{\delta}\left\|\frac{d}{d s}\left(\varphi_{\tau, u} \circ \gamma(s)\right)\right\| d s=\int_{0}^{\delta}\left\|D \varphi_{\tau, u}(\gamma(s)) \dot{\gamma}(s)\right\| d s .
\end{aligned}
$$

Hence,

$$
C \mathrm{e}^{\lambda \tau} \leq \frac{1}{\delta} \int_{0}^{\delta}\left\|D \varphi_{\tau, u}(\gamma(s)) \dot{\gamma}(s)\right\| d s \leq \sup _{s \in[0, \delta]}\left\|D \varphi_{\tau, u}(\gamma(s)) \dot{\gamma}(s)\right\|
$$


For $\delta \searrow 0$ this implies $\left\|D \varphi_{\tau, u}\left(x_{1}\right) v\right\| \geq C \mathrm{e}^{\lambda \tau}=C \mathrm{e}^{\lambda \tau}\|v\|$. Therefore, we obtain

$$
\inf _{x \in Q} \omega_{n}^{E}(u, x, \tau)=\inf _{x \in Q} \operatorname{det} D \varphi_{\tau, u}(x) \geq\left(C \mathrm{e}^{\lambda \tau}\right)^{m} \rightarrow \infty .
$$

This proves the claim. The right-hand side of $(23)$ is positive, which is shown as follows (using the Liouville formula and (24)):

$$
\begin{aligned}
\limsup _{\tau \rightarrow \infty} \frac{1}{\tau} \inf _{(u, x) \in \mathcal{K}_{Q}} \int_{0}^{\tau} \operatorname{div} F_{u(s)}\left(\varphi_{s, u}(x)\right) d s & =\limsup _{\tau \rightarrow \infty} \frac{1}{\tau} \inf _{(u, x) \in \mathcal{K}_{Q}} \ln \operatorname{det} D \varphi_{\tau, u}(x) \\
& \geq \limsup _{\tau \rightarrow \infty} \frac{1}{\tau} \ln \left(C \mathrm{e}^{\lambda \tau}\right)^{d}=d \lambda>0 .
\end{aligned}
$$

This finishes the proof.

Remark 5.3 By replacing the integrand in $(23)$ by $\min _{(x, u) \in Q \times U} \operatorname{div} F_{u}(x)$ we recover the earlier estimate

$$
h_{\text {inv }}^{*}(K, Q) \geq \min _{(x, u) \in Q \times U} \operatorname{div} F_{u}(x),
$$

which also holds for $h_{\text {inv }}(K, Q)$ and without the assumption of uniform expansiveness.

\section{Inhomogeneous bilinear systems}

Consider the inhomogeneous bilinear system

$$
\dot{x}(t)=\left[A_{0}+\sum_{i=1}^{m_{1}} u_{i}(t) A_{i}\right] x(t)+B v(t), \quad(u, v) \in \mathcal{U} \times \mathcal{V},
$$

on $\mathbb{R}^{d}$. Assume that the control range is the product of two compact and convex sets $U \subset \mathbb{R}^{m_{1}}$ and $V \subset \mathbb{R}^{m_{2}}\left(m_{1}+m_{2}=m\right)$. For each $u \in \mathcal{U}$ let $\Lambda_{u}(t, s)$ be the evolution operator of the homogeneous equation

$$
\dot{x}(t)=\left[A_{0}+\sum_{i=1}^{m_{1}} u_{i}(t) A_{i}\right] x(t) .
$$

We also write $A(u):=A_{0}+\sum_{i=1}^{m_{1}} u_{i} A_{i}$. By the variation of constants formula, the solution of 25 can be written as

$$
\varphi(t, x,(u, v))=\Lambda_{u}(t, 0) x+\int_{0}^{t} \Lambda_{u}(t, s) B v(s) d s .
$$

Recall that for a control-affine system (so in particular for an inhomogeneous bilinear system) with compact and convex control range the set of admissible control functions becomes a compact metrizable space when endowed with the weak*-topology of $L^{\infty}\left(\mathbb{R}, \mathbb{R}^{m}\right)=L^{1}\left(\mathbb{R}, \mathbb{R}^{m}\right)^{*}$, and that both the shift flow and the cocycle $\varphi(t, x, u)$ are continuous mappings with respect to this topology. The mappings $\Phi_{t}=\left(\Theta_{t}, \varphi(t, \cdot, \cdot)\right)$, $t \in \mathbb{R}$, define a continuous skew-product flow, the so-called control flow of the system. 
Lower bounds for the strict invariance entropy

Proposition 6.1 Let $Q$ be a compact controlled invariant set for system (25) and let $K \subset Q$ be compact. Assume that there exists a vector bundle decomposition

$$
\mathcal{U} \times \mathbb{R}^{d}=\mathcal{W}^{+} \oplus \mathcal{W}^{-}, \quad d^{ \pm}:=\operatorname{Rank} \mathcal{W}^{ \pm},
$$

into subbundles $\mathcal{W}^{+}$and $\mathcal{W}^{-}$, respectively, both invariant under the control flow of the homogeneous bilinear control system corresponding to (25) such that the following holds: There are constants $C, \lambda>0$ with

$$
\left\|\Lambda_{u}(t, 0) x\right\| \geq C \mathrm{e}^{\lambda t}\|x\| \quad \text { for all } t \geq 0,(u, x) \in \mathcal{W}^{+},
$$

and for every $\delta>0$ there is $D>0$ with

$$
\left\|\Lambda_{u}(t, 0) x\right\| \leq D \mathrm{e}^{\delta t}\|x\| \quad \text { for all } t \geq 0,(u, x) \in \mathcal{W}^{-} .
$$

Then $h_{\mathrm{esc}}(K, Q) \leq 0$.

Proof. Let $\pi^{+}(u)$ and $\pi^{-}(u)$ denote the corresponding projections onto $W^{+}(u)$ and $W^{-}(u)(u \in \mathcal{U})$. Then

$$
\pi^{ \pm}\left(\Theta_{t} u\right) \Lambda_{u}(t, 0)=\Lambda_{u}(t, 0) \pi^{ \pm}(u) \text { for all } t \in \mathbb{R}, u \in \mathcal{U}
$$

For arbitrary $(u, v) \in \pi_{\mathcal{U} \times \mathcal{V}} \mathcal{K}_{Q}, \tau>0$, and $\varepsilon>0$ let $S$ be a $((u, v), \tau, \varepsilon)$-separated subset of $K((u, v), \tau)$. From the solution formula $(26)$ it follows that

$$
\varphi(t, x,(u, v))-\varphi(t, y,(u, v)) \equiv \Lambda_{u}(t, 0)(x-y),
$$

and hence for each pair of distinct elements $x, y \in S$ we obtain

$$
\begin{aligned}
\varepsilon & \leq \max _{t \in[0, \tau]}\left\|\Lambda_{u}(t, 0)(x-y)\right\| \\
& =\max _{t \in[0, \tau]}\left\|\pi^{+}\left(\Theta_{t} u\right) \Lambda_{u}(t, 0)(x-y)+\pi^{-}\left(\Theta_{t} u\right) \Lambda_{u}(t, 0)(x-y)\right\| \\
& \leq \max _{t \in[0, \tau]}\left(\left\|\pi^{+}\left(\Theta_{t} u\right) \Lambda_{u}(t, 0)(x-y)\right\|+\left\|\pi^{-}\left(\Theta_{t} u\right) \Lambda_{u}(t, 0)(x-y)\right\|\right) \\
& \leq \max _{t \in[0, \tau]}\left\|\pi^{+}\left(\Theta_{t} u\right) \Lambda_{u}(t, 0)(x-y)\right\|+\max _{t \in[0, \tau]}\left\|\pi^{-}\left(\Theta_{t} u\right) \Lambda_{u}(t, 0)(x-y)\right\| \\
& \stackrel{29}{=} \max _{t \in[0, \tau]}\left\|\Lambda_{u}(t, 0) \pi^{+}(u)(x-y)\right\|+\max _{t \in[0, \tau]}\left\|\Lambda_{u}(t, 0) \pi^{-}(u)(x-y)\right\| .
\end{aligned}
$$

Assume that the first maximum in the last term is attained at $s^{+} \in[0, \tau]$ and the second one at $s^{-} \in[0, \tau]$. Then, using the cocycle property of the solution, we get

$$
\begin{aligned}
\left\|\Lambda_{u}(\tau, 0) \pi^{+}(u)(x-y)\right\| & =\left\|\Lambda_{\Theta_{s^{+}}}\left(\tau-s^{+}, 0\right) \Lambda_{u}\left(s^{+}, 0\right) \pi^{+}(u)(x-y)\right\| \\
& \stackrel{277}{\geq} C \mathrm{e}^{\lambda\left(\tau-s^{+}\right)}\left\|\Lambda_{u}\left(s^{+}, 0\right) \pi^{+}(u)(x-y)\right\| \\
& \geq C \max _{t \in[0, \tau]}\left\|\Lambda_{u}(t, 0) \pi^{+}(u)(x-y)\right\|
\end{aligned}
$$

and

$$
\begin{aligned}
\max _{t \in[0, \tau]}\left\|\Lambda_{u}(t, 0) \pi^{-}(u)(x-y)\right\| & =\left\|\Lambda_{u}\left(s^{-}, 0\right) \pi^{-}(u)(x-y)\right\| \\
& \stackrel{288}{\leq} D \mathrm{e}^{\delta s^{-}}\left\|\pi^{-}(u)(x-y)\right\| \\
& \leq D \mathrm{e}^{\delta \tau}\left\|\pi^{-}(u)(x-y)\right\| .
\end{aligned}
$$


Hence, altogether we obtain

$$
\varepsilon \leq C^{-1}\left\|\Lambda_{u}(\tau, 0) \pi^{+}(u)(x-y)\right\|+D \mathrm{e}^{\delta \tau}\left\|\pi^{-}(u)(x-y)\right\| .
$$

With $\gamma=\gamma(\tau):=\min \left\{C, D^{-1} \mathrm{e}^{-\delta \tau}\right\}$ this gives

$$
\left\|\Lambda_{u}(\tau, 0) \pi^{+}(u)(x-y)\right\|+\left\|\pi^{-}(u)(x-y)\right\| \geq \gamma \varepsilon,
$$

which implies

$$
\left\|\Lambda_{u}(\tau, 0) \pi^{+}(u)(x-y)\right\| \geq \frac{\gamma \varepsilon}{2} \text { or } \quad\left\|\pi^{-}(u)(x-y)\right\| \geq \frac{\gamma \varepsilon}{2} .
$$

Now we cover $Q$ with sets $Q_{1}, \ldots, Q_{n}$ such that for each $i \in\{1, \ldots, n\}$ and $x, y \in S \cap Q_{i}$ it holds that $\left\|\pi^{-}(u)(x-y)\right\|<(\gamma \varepsilon) / 2$. The sets $Q_{1}, \ldots, Q_{n}$ can be defined as follows: First cover $\pi^{-}(u) Q$ with a minimal collection of $d^{-}$-dimensional balls of radii $(\gamma \varepsilon) / 2$, say $B_{1}, \ldots, B_{n}, n=n(u, \varepsilon, \tau)$, and then define

$$
Q_{i}:=\pi^{+}(u) Q \oplus B_{i}, \quad i=1, \ldots, n .
$$

Then for each distinct $x, y \in S \cap Q_{i}$ it must hold that $\left\|\Lambda_{u}(\tau, 0) \pi^{+}(u)(x-y)\right\| \geq(\gamma \varepsilon) / 2$, so in particular $\pi^{+}(u) x \neq \pi^{+}(u) y$. Hence, the set $\Lambda_{u}(\tau, 0) \pi^{+}(u)\left(S \cap Q_{i}\right)$ is $(\gamma \varepsilon) / 2$ separated and has the same cardinality as $S \cap Q_{i}$. Using that $S \subset K(u, \tau)$ we obtain

$$
\begin{aligned}
\Lambda_{u}(\tau, 0) \pi^{+}(u)\left(S \cap Q_{i}\right) & =\pi^{+}\left(\Theta_{\tau} u\right) \Lambda_{u}(\tau, 0)\left(S \cap Q_{i}\right) \\
& \stackrel{26]}{\subset} \pi^{+}\left(\Theta_{\tau} u\right)\left[\varphi\left(\tau, S \cap Q_{i},(u, v)\right)-\int_{0}^{\tau} \Lambda_{u}(t, s) B v(s) d s\right] \\
& \subset \pi^{+}\left(\Theta_{\tau} u\right) Q-\underbrace{\pi^{+}\left(\Theta_{\tau} u\right) \int_{0}^{\tau} \Lambda_{u}(t, s) B v(s) d s}_{=: b(u, v, \tau)} .
\end{aligned}
$$

Since $\pi^{+}(\cdot)$ is continuous and $\mathcal{U}$ is compact in the weak*-topology, there is $R>0$ such that each of the sets $\pi^{+}\left(\Theta_{\tau} u\right) Q-b(u, v, \tau)$ is contained in a $d^{+}$-dimensional ball with radius $R$ contained in $W^{+}(u)$. Using a volume argument, one easily sees that the maximal cardinality of a $(\gamma \varepsilon) / 2$-separated set contained in such a ball is approximately $((2 R) /(\gamma \varepsilon))^{d^{+}}$. Also $\pi^{-}(\cdot)$ is continuous and hence there is $r>0$ such that $\pi^{-}(u) Q$ is contained in a $d^{-}$-dimensional ball with radius $r$ centred at $0 \in W^{-}(u)$, which implies that $n$ is approximately $((2 r) /(\gamma \varepsilon))^{d_{-}}$. We thus obtain

$$
\begin{aligned}
\# S & \leq \sum_{i=1}^{n} \#\left(S \cap Q_{i}\right)=\sum_{i=1}^{n} \# \Lambda_{u}(\tau, 0) \pi^{+}(u)\left(S \cap Q_{i}\right) \\
& \leq\left(\frac{2 r}{\gamma \varepsilon}\right)^{d^{-}}\left(\frac{2 R}{\gamma \varepsilon}\right)^{d^{+}}=\frac{\mathrm{const}}{\gamma(\tau)^{d} \varepsilon^{d}} .
\end{aligned}
$$

This implies $\bar{r}_{\text {sep }}(\tau, K, Q) \leq$ const $\cdot \gamma(\tau)^{-d}=$ const $\cdot \min \left\{C, D^{-1} \mathrm{e}^{-\delta \tau}\right\}^{-d}$ and hence

$$
h_{\mathrm{esc}}(K, Q) \leq \limsup _{\tau \rightarrow \infty} \frac{1}{\tau} \ln \left(D^{d} \mathrm{e}^{\delta d \tau}\right)=\delta d .
$$

Since $\delta$ can be chosen arbitrarily small, we obtain $h_{\mathrm{esc}}(K, Q) \leq 0$. 
Corollary 6.2 In addition to the assumptions of the preceding proposition, suppose that $K$ has positive Lebesgue measure and $h_{\mathrm{inv}}^{*}(Q)<\infty$. Then

$$
h_{\mathrm{inv}}^{*}(K, Q) \geq \limsup _{\tau \rightarrow \infty} \frac{1}{\tau} \inf _{u \in \pi_{\mathcal{U}} \mathcal{K}_{Q}} \int_{0}^{\tau} \operatorname{tr}\left[A(u(s)) \circ Q\left(\Theta_{s} u\right)\right] d s,
$$

where $Q(u): \mathbb{R}^{d} \rightarrow W^{+}(u)$ is the orthogonal projection, and $\pi_{\mathcal{U}}: \mathcal{U} \times \mathcal{V} \times \mathbb{R}^{m} \rightarrow \mathcal{U}$, $(u, v, x) \mapsto u$. In particular, if the subbundle $\mathcal{W}^{+}$is nontrivial, then $h_{\mathrm{inv}}^{*}(K, Q)>0$.

Proof. Define a subbundle $E$ of $\bigcup_{((u, v), x) \in \mathcal{Q}}\{(u, v)\} \times T_{x} M$ by $E_{(u, v), x}:=W^{+}(u)$ for all $((u, v), x) \in \mathcal{Q}$. Then combine Corollary 4.2 and Proposition 6.1. To show that $h_{\text {inv }}^{*}(K, Q)>0$ if $d^{+}>0$, note that

$$
\begin{aligned}
\limsup _{\tau \rightarrow \infty} \frac{1}{\tau} \inf _{u} \int_{0}^{\tau} \operatorname{tr}\left[A(u(s)) \circ Q\left(\Theta_{s} u\right)\right] d s & =\left.\limsup _{\tau \rightarrow \infty} \frac{1}{\tau} \inf _{u} \ln \operatorname{det} \Lambda_{u}(\tau, 0)\right|_{W^{+}(u)} \\
& \geq \limsup _{\tau \rightarrow \infty} \frac{1}{\tau} \ln \left(C \mathrm{e}^{\lambda \tau}\right)^{d_{+}}=\lambda d^{+}>0 .
\end{aligned}
$$

This finishes the proof.

The following theorem shows that the estimate (30) also holds without the assumption on the subbundles in Proposition 6.1, which in general is a restrictive condition. The proof is not based on our main theorem, Theorem 4.1, but uses similar techniques. In particular, it uses the following lemma which can be found in [9, Lem. 4.1.8]:

Lemma 6.3 Let $k \in \mathbb{N}, K \subset \mathbb{R}^{d}$ a compact set with positive Lebesgue measure and $\mathcal{P} \subset \mathbb{R}^{d \times d}$ a compact set of projections with $k$-dimensional image. For all $P \in \mathcal{P}$ let $\lambda_{P}^{k}$ denote the $k$-dimensional Lebesgue measure in $\operatorname{im}(P)$. Then there exists $\alpha>0$ such that for every finite open covering $\left\{K_{1}, \ldots, K_{r}\right\}$ of the set $K$ (i.e., $K=\bigcup_{j=1}^{r} K_{j}$ and the sets $K_{j}$ are open relative to $K$ ) and for all $P_{1}, \ldots, P_{r} \in \mathcal{P}$ we have

$$
\sum_{j=1}^{r} \lambda_{P_{j}}^{k}\left(P_{j}\left(K_{j}\right)\right) \geq \alpha
$$

Theorem 6.4 Consider the inhomogeneous bilinear system (25). Let $Q \subset \mathbb{R}^{d}$ be a compact controlled invariant set with $h_{\mathrm{inv}}^{*}(Q)<\infty$, and $K \subset Q$ a compact set with positive Lebesgue measure. Then there exists a maximal invariant subbundle $\mathcal{W}^{+} \subset \mathcal{U} \times \mathbb{R}^{d}$ on which the system is uniformly expanding such that the estimate

$$
h_{\text {inv }}^{*}(K, Q) \geq \limsup _{\tau \rightarrow \infty} \inf _{u \in \pi_{\mathcal{U}} \mathcal{K}_{Q}} \frac{1}{\tau} \int_{0}^{\tau} \operatorname{tr}\left[A(u(s)) \circ Q\left(\Theta_{s} u\right)\right] d s
$$

holds with $Q(u)$ and $\pi_{\mathcal{U}}$ as in Corollary 6.2.

Proof. We prove the theorem in two steps.

Step 1: Selgrade's Theorem (see, e.g, [3, Theo. 5.2.5]) yields a decomposition $\mathcal{U} \times \overline{\mathbb{R}}^{d}=\mathcal{W}_{1} \oplus \cdots \oplus \mathcal{W}_{r}$ into subbundles, which are invariant under the control flow of the homogeneous system corresponding to $(25)$. We can define $\mathcal{W}^{+}$as the Whitney 
sum of all those subbundles on which the system is uniformly expanding. Then there are $C, \lambda>0$ such that

$$
\left\|\Lambda_{u}(t, 0) x\right\| \geq C \mathrm{e}^{\lambda t}\|x\| \text { for all } t \geq 0, \quad(u, x) \in \mathcal{W}^{+} .
$$

Let $\mathcal{W}^{-}$be the sum of the remaining subbundles. For each $u \in \mathcal{U}$, let $\pi_{u}^{+}: \mathbb{R}^{d} \rightarrow W^{+}(u)$ be the projection onto $W^{+}(u)$ with respect to the decomposition $\mathcal{U} \times \mathbb{R}^{d}=\mathcal{W}^{+} \oplus \mathcal{W}^{-}$, and let $d^{+}$be the rank of $\mathcal{W}^{+}$. By $m^{d_{+}}$we denote the $d^{+}$-dimensional Lebesgue measure on any of the fibres $W^{+}(u), u \in \mathcal{U}$. Let $\mathcal{S} \subset \pi_{\mathcal{U} \times \mathcal{V}} \mathcal{K}_{Q}$ be a minimal $(\tau, K, Q)$-spanning set. From the assumption $h_{\text {inv }}^{*}(Q)<\infty$ finiteness of $\mathcal{S}$ follows. Moreover,

$$
K=\bigcup_{(u, v) \in \mathcal{S}} K((u, v), \tau) .
$$

By Lemma 6.3 there is a constant $\alpha>0$ (only depending on $K$ ) with

$$
\begin{aligned}
\alpha & \leq \sum_{(u, v) \in \mathcal{S}} m^{d_{+}}\left(\pi_{u}^{+}(K((u, v), \tau))\right) \\
& \leq \# \mathcal{S} \cdot \sup _{(u, v) \in \pi_{\mathcal{U} \times \mathcal{V} \mathcal{K}_{Q}}} m^{d_{+}}\left(\pi_{u}^{+}(K((u, v), \tau))\right) .
\end{aligned}
$$

Since $\# \mathcal{S}=r_{\text {inv }}^{*}(\tau, K, Q)$, this implies

$$
\begin{aligned}
h_{\mathrm{inv}}^{*}(K, Q) & \geq \limsup _{\tau \rightarrow \infty} \frac{1}{\tau} \ln \frac{\alpha}{\sup _{(u, v)} m^{d_{+}}\left(\pi_{u}^{+}(K((u, v), \tau))\right)} \\
& =\limsup _{\tau \rightarrow \infty}\left[-\frac{1}{\tau} \ln \sup _{(u, v) \in \pi_{\mathcal{U} \times \mathcal{V}} \mathcal{K}_{Q}} m^{d_{+}}\left(\pi_{u}^{+}(K((u, v), \tau))\right)\right] .
\end{aligned}
$$

Step 2: Fix an arbitrary $\varepsilon>0$. For each $\tau>0$ and $(u, v) \in \pi_{\mathcal{U} \times \mathcal{V}} \mathcal{K}_{Q}$ select a maximal $((u, v), \tau, \varepsilon)$-separated set $S_{(u, v), \tau} \subset \pi_{u}^{+}(K((u, v), \tau)) \subset W^{+}(u)$. Then for all distinct $x_{1}, x_{2} \in S_{(u, v), \tau}$ it holds that

$$
\max _{t \in[0, \tau]}\left\|\Lambda_{u}(t, 0)\left(x_{1}-x_{2}\right)\right\|=\max _{t \in[0, \tau]}\left\|\varphi\left(t, x_{1},(u, v)\right)-\varphi\left(t, x_{2},(u, v)\right)\right\| \geq \varepsilon .
$$

Since $x_{1}-x_{2} \in W^{+}(u)$, from 32 we obtain (with the same arguments as in the proof of Proposition 5.1)

$$
\left\|\Lambda_{u}(\tau, 0)\left(x_{1}-x_{2}\right)\right\| \geq C \varepsilon .
$$

The set $\Lambda_{u}(\tau, 0) S_{(u, v), \tau}$ has the same cardinality as $S_{(u, v), \tau}$ and

$$
\begin{aligned}
S_{(u, v), \tau} & \subset\left\{\pi_{u}^{+} x: \varphi(\tau, x,(u, v)) \in Q\right\} \\
& =\left\{\pi_{u}^{+} x: \Lambda_{u}(\tau, 0) x \in Q-\varphi^{s}(\tau, u, v)\right\} .
\end{aligned}
$$

Hence,

$$
\begin{aligned}
\Lambda_{u}(\tau, 0) S_{(u, v), \tau} & \subset\left\{\Lambda_{u}(\tau, 0) \pi_{u}^{+} x: \Lambda_{u}(\tau, 0) x \in Q-\varphi^{s}(\tau, u, v)\right\} \\
& =\left\{\pi_{\Theta_{\tau} u}^{+} \Lambda_{u}(\tau, 0) x: \Lambda_{u}(\tau, 0) x \in Q-\varphi^{s}(\tau, u, v)\right\} \\
& =\left\{\pi_{\Theta_{\tau} u}^{+} y: y \in Q-\varphi^{s}(\tau, u, v)\right\} \\
& =\pi_{\Theta_{\tau} u}^{+} Q-\pi_{\Theta_{\tau} u}^{+} \varphi^{s}(\tau, u, v) .
\end{aligned}
$$


The projection $\pi_{u}^{+}$depends continuously on $u$ in the weak*-topology and hence, by compactness of $\mathcal{U}$, there is $R>0$ such that each of the sets $\pi_{u}^{+} Q, u \in \mathcal{U}$, is contained in a ball in $W^{+}(u)$ of radius $R$ centred at the origin. Therefore,

$$
\Lambda_{u}(\tau, 0) S_{(u, v), \tau}+\pi_{\Theta_{\tau} u}^{+} \varphi^{s}(\tau, u, v) \subset B_{R}(0) \subset W^{+}(u) .
$$

The set on the left-hand side has the same cardinality as $S_{(u, v), \tau}$ and each two distinct points in this set have distance $\geq C \varepsilon$. This implies that there is a global bound $N \in \mathbb{N}$ on $\# S_{(u, v), \tau}$, i.e.,

$$
\# S_{(u, v), \tau} \leq N \text { for all }(u, v) \in \pi_{\mathcal{U} \times \mathcal{V}}, \tau>0 .
$$

Since $S_{(u, v), \tau}$ is maximal $((u, v), \tau, \varepsilon)$-separated in $\pi_{u}^{+}(K((u, v), \tau))$, we have

$$
\pi_{u}^{+}(K((u, v), \tau)) \subset \bigcup_{x \in S_{(u, v), \tau}} B_{\varepsilon}^{\tau,+}((u, v), x),
$$

where

$$
B_{\varepsilon}^{\tau,+}((u, v), x):=B_{\varepsilon}^{\tau}((u, v), x) \cap W^{+}(u) .
$$

Hence,

$$
\begin{aligned}
m^{d_{+}}\left(\pi_{u}^{+}(K((u, v), \tau))\right) & \leq \sum_{x \in S_{(u, v), \tau}} m^{d_{+}}\left(B_{\varepsilon}^{\tau,+}((u, v), x)\right) \\
& \leq \# S_{(u, v), \tau} \cdot \max _{x} m^{d_{+}}\left(B_{\varepsilon}^{\tau,+}((u, v), x)\right) \\
& \leq 23 \cdot \max _{x} m^{d_{+}}\left(B_{\varepsilon}^{\tau,+}((u, v), x)\right) .
\end{aligned}
$$

We have

$$
\begin{aligned}
B_{\varepsilon}^{\tau,+}((u, v), x) & =\left\{y \in W^{+}(u): \max _{t \in[0, \tau]}\left\|\Lambda_{u}(t, 0)(x-y)\right\|<\varepsilon\right\} \\
& \subset\left\{y \in W^{+}(u):\left\|\Lambda_{u}(\tau, 0)(x-y)\right\|<\varepsilon\right\} .
\end{aligned}
$$

Since Lebesgue measure is invariant under translations, this implies

$$
\begin{aligned}
m^{d_{+}}\left(B_{\varepsilon}^{\tau,+}((u, v), x)\right) & \leq m^{d_{+}}\left(\left\{y \in W^{+}(u):\left\|\Lambda_{u}(\tau, 0) y\right\|<\varepsilon\right\}\right) \\
& \leq m^{d_{+}}\left(\Lambda_{u}(\tau, 0)^{-1}\left(B_{\varepsilon}(0) \cap W^{+}\left(\Theta_{\tau} u\right)\right)\right) \\
& =C(\varepsilon)\left(\left.\operatorname{det} \Lambda_{u}(\tau, 0)\right|_{W^{+}(u)}\right)^{-1},
\end{aligned}
$$

where $C(\varepsilon)$ is the volume of the $d^{+}$-dimensional Euclidean $\varepsilon$-ball. Thus, with Step 1 we obtain

$$
\begin{aligned}
h_{\text {inv }}^{*}(K, Q) & \geq \limsup _{\tau \rightarrow \infty}\left[-\frac{1}{\tau} \ln \sup _{(u, v) \in \pi_{\mathcal{U} \times \mathcal{V}} \mathcal{K}_{Q}} m^{d_{+}}\left(\pi_{u}^{+}(K((u, v), \tau))\right)\right] \\
& \geq \limsup _{\tau \rightarrow \infty}\left[-\frac{1}{\tau} \ln \sup _{(u, v) \in \pi \mathcal{U} \times \mathcal{V} \mathcal{K}_{Q}} N \cdot \max _{x} m^{d_{+}}\left(B_{\varepsilon}^{\tau,+}((u, v), x)\right)\right] \\
& \geq \limsup _{\tau \rightarrow \infty}\left[-\frac{1}{\tau} \ln \sup _{(u, v) \in \pi_{\mathcal{U} \times \mathcal{V}} \mathcal{K}_{Q}} N C(\varepsilon)\left(\left.\operatorname{det} \Lambda_{u}(\tau, 0)\right|_{W^{+}(u)}\right)^{-1}\right]
\end{aligned}
$$




$$
\begin{aligned}
& =\limsup _{\tau \rightarrow \infty}\left[-\frac{1}{\tau} \ln \sup _{u \in \pi_{\mathcal{U}} \mathcal{K}_{Q}}\left(\left.\operatorname{det} \Lambda_{u}(\tau, 0)\right|_{W^{+}(u)}\right)^{-1}\right] \\
& =\left.\limsup _{\tau \rightarrow \infty} \inf _{u \in \pi_{\mathcal{U}} \mathcal{K}_{Q}} \frac{1}{\tau} \ln \operatorname{det} \Lambda_{u}(\tau, 0)\right|_{W^{+}(u)} .
\end{aligned}
$$

From invariance of $\mathcal{W}^{+}$it follows as in the proof of Corollary 4.2 that

$$
\left.\operatorname{det} \Lambda_{u}(\tau, 0)\right|_{W^{+}(u)}=\exp \left(\int_{0}^{\tau} \operatorname{tr}\left[A(u(s)) \circ Q\left(\Theta_{s} u\right)\right] d s\right) \text {. }
$$

This finishes the proof.

Remark 6.5 For a linear control system

$$
\dot{x}(t)=A x(t)+B u(t), \quad u \in \mathcal{U},
$$

estimate (31) yields

$$
h_{\mathrm{inv}}^{*}(K, Q) \geq \sum_{\lambda \in \sigma(A)} \max \{0, \operatorname{Re}(\lambda)\}
$$

when we define the subbundle $\mathcal{W}^{+}$by $W^{+}(u): \equiv \mathbb{E}^{u}(A)$ (the unstable subspace associated with $A$ ) and $\mathcal{W}^{-}$by $W^{-}(u): \equiv \mathbb{E}^{s c}(A)$ (the centre-stable subspace). This is the exact value of $h_{\text {inv }}(K, Q)$ (not necessarily $h_{\text {inv }}^{*}(K, Q)$ ), as was proved in [2, Theo. $5.1]$.

\section{Open questions and future directions}

We end with some conjectures and remarks.

\section{Remark 7.1}

(i) We conjecture that $h_{\mathrm{esc}}(K, Q) \geq 0$ if $\mu_{C}(K, d$; $\varrho)>0$.

(ii) One could try to drop the assumption of $K$ having positive $d$-dimensional Hausdorff measure in Theorem 4.1. We conjecture that the estimate (18) then still holds in a slightly modified version.

(iii) We conjecture that in general $h_{\mathrm{esc}}(K, Q)$ is completely determined by the behaviour of the systems $\left(\varphi_{t, u}\right)_{t \in \mathbb{R}}, u \in \pi_{\mathcal{U}} \mathcal{K}_{Q}$, on the sets

$$
K_{\infty}(u)=\left\{x \in K: \varphi\left(\mathbb{R}_{0}^{+}, x, u\right) \subset Q\right\} .
$$

(iv) Certainly, similar lower estimates for $h_{\text {inv }}(K, Q)$ can be derived, whereas things get technically more complicated here. In fact, that is the reason why we considered the quantity $h_{\text {inv }}^{*}(K, Q)$ rather than $h_{\text {inv }}(K, Q)$.

\section{Acknowledgments}

This work was supported by DFG grant Co 124/17-1 within DFG priority program 1305. 


\section{References}

[1] Boichenko V A, Leonov G A and Reitmann V 2005 Dimension Theory for Ordinary Differential Equations (Wiesbaden: Teubner)

[2] Colonius F and Kawan C 2009 Invariance entropy for control systems SIAM J. Control Optim. 48 1701-21

[3] Colonius F and Kliemann W 2000 The Dynamics of Control (Boston: Birkhäuser)

[4] Douady A and Oesterle J 1980 Dimension de Hausdorff des attracteurs C. R. Acad. Sci. Paris, Sér. A 290 1135-38

[5] Franz A R 1998 Hausdorff dimension estimates for invariant sets with an equivariant tangent bundle splitting Nonlinearity 11 1063-74

[6] Gelfert K 2001 Abschätzungen der kapazitiven Dimension und der topologischen Entropie für partiell volumenexpandierende sowie volumenkontrahierende Systeme auf Mannigfaltigkeiten (PhD Thesis, Technical University of Dresden)

[7] Gelfert K 2005 Lower bounds for the topological entropy Discrete Contin. Dyn. Syst. 12 555-65

[8] Kawan C 2009 Upper and lower estimates for invariance entropy, submitted

[9] Kawan C 2010 Invariance Entropy for Control Systems (PhD Thesis, University of Augsburg)

[10] Kawan C 2010 Invariance entropy of control sets, submitted

[11] Kolyada S and Snoha L 1996 Topological entropy of nonautonomous dynamical systems Random Comput. Dyn. 4 205-33

[12] Temam R 1988 Infinite-Dimensional Dynamical Systems in Mechanics and Physics (New York: Springer)

[13] Young L-S 1990 Some large deviation results for dynamical systems Trans. Am. Math. Soc. 318 $525-43$

[14] Young L-S and Demers M F 2006 Escape rates and conditionally invariant measures Nonlinearity $19377-79$ 